\title{
Supporting Information: Nonadiabatic Dynamics Simulations on Early-Time Photochemistry of Spirobenzopyran
}

\author{
Ya-Hui Zhang, Xin-Wei Sun, Teng-Shuo Zhang, Xiang-Yang Liu*, and Ganglong Cui* \\ Key Laboratory of Theoretical and Computational Photochemistry, Ministry of Education, College of \\ Chemistry, Beijing Normal University, Beijing 100875, China \\ E-mail: xiangyangliu@sicnu.edu.cn; ganglong.cui@bnu.edu.cn
}

\section{Table of Contents}

\section{I . Figures}

1. Figure S1: molecular orbitals relevant to electronic transitions of SPc.

2. Figure S2: molecular orbitals relevant to electronic transitions of SPt.

3. Figure S3: molecular orbitals relevant to electronic transitions of SPmc.

4. Figure S4: molecular orbitals relevant to electronic transitions of SPmt.

5. Figure S5: energy profile of ground-state conversion from SPc to SPt.

6. Figure S6: overlap of OM2MRCl and CASSCF optimized structures.

7. Figure S7: two-dimensional potential energy surfaces.

8. Figure S8: photochromic mechanism of SPt.

9. Figure S9: two typical trajectories for SPc.

10. Figure S10: two typical trajectories for SPc.

11. Figure $\mathbf{S 1 1}$ : geometry parameter distributions at hopping points.

12. Figure S12: optimized structures of spirobenzopyran (SPm).

13. Figure S13: optimized ground-state equilibrium structures of merocyanine (MC).

14. Figure S14: distributions of selected geometric parameters and hopping times of SPm.

15. Figure S15: photochromic mechanism of SPmc.

16. Figure S16: photochromic mechanism of SPmt.

17. Figure S17: two typical trajectories for SPt.

18. Figure S18: three typical trajectories for SPt.

19. Figure S19: three typical trajectories for SPt.

20. Figure S20: two typical trajectories for SPt.

\section{Tables}

21. Table S1: computed relative energies of SPh, MC forms and MECls.

22. Table S2: computed energies, oscillator strengths, and electronic configurations of SPc.

23. Table S3: computed energies, oscillator strengths, and electronic configurations of SPt.

24. Table S4: computed energies, oscillator strengths, and electronic configurations of SPmc.

25. Table S5: computed energies, oscillator strengths, and electronic configurations of SPmt.

III. Cartesian coordinates 


\section{I . Figures}

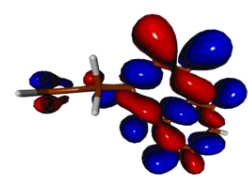

HOMO-4

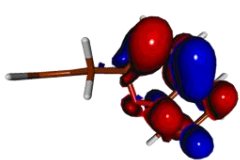

LUMO

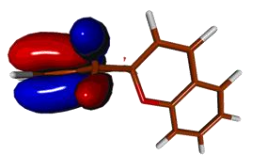

HOMO-3

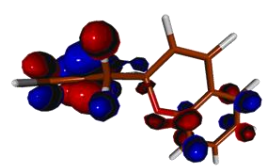

LUMO+1

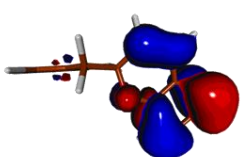

HOMO-2

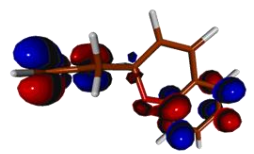

LUMO+2

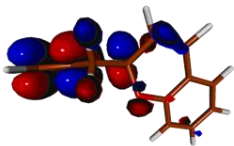

HOMO-1

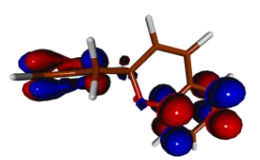

LUMO+3

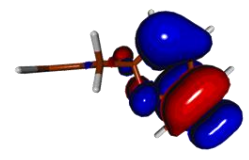

HOMO

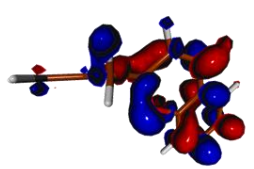

LUMO+4

Fig. S1: OM2/MRCI computed molecular orbitals used as the active space for relevant electronic transitions of SPc.

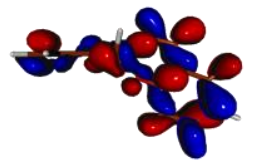

HOMO-4

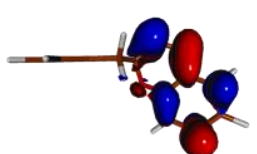

LUMO

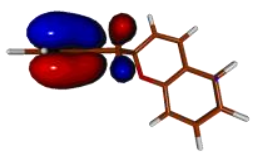

номо-3

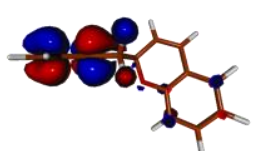

LUMO+1

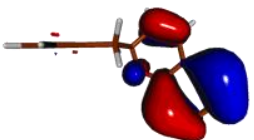

HOMO-2

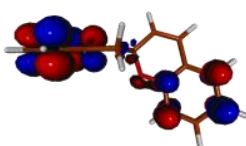

LUMO+2

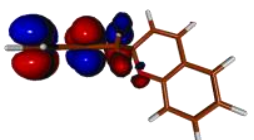

HOMO-1

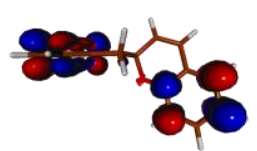

LUMO+3

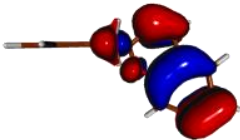

HOMO

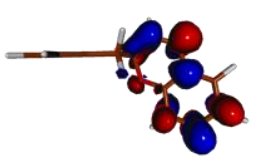

LUMO+4

Fig. S2: OM2/MRCI computed molecular orbitals used as the active space for relevant electronic transitions of SPt.

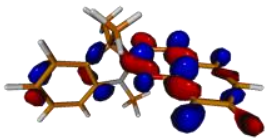

HOMO-4

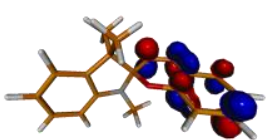

LUMO

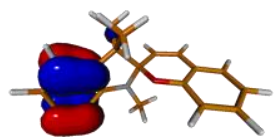

HOMO-3

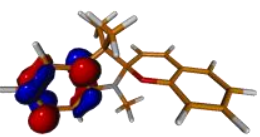

LUMO+1

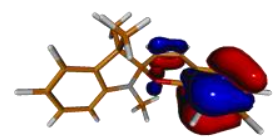

HOMO-2

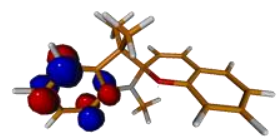

LUMO+2

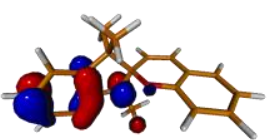

HOMO-1

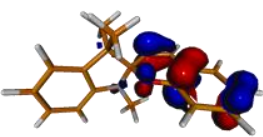

HOMO

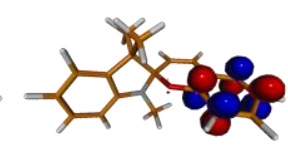

LUMO+3

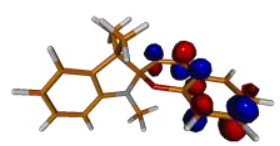

LUMO+4

Fig. S3: OM2/MRCI computed molecular orbitals used as the active space for relevant electronic transitions of SPmc. 

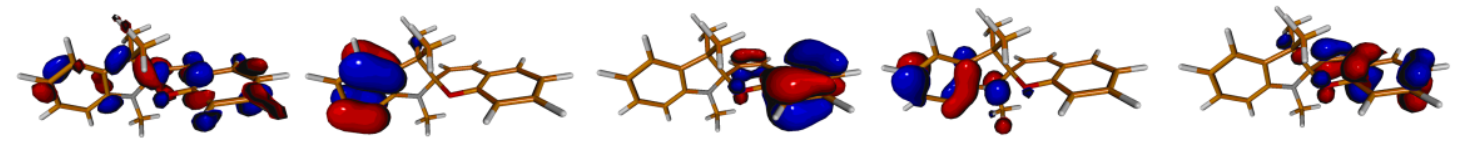

номо-4

номо-3

Hомо-2

HOMO-1

номо
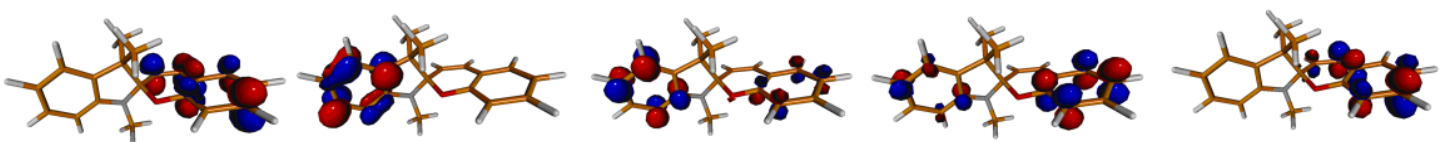

LUMO

LUMO+1

LUMO+2

LUMO+3

LUMO+4

Fig. S4: OM2/MRCI computed molecular orbitals used as the active space for relevant electronic transitions of SPmt.

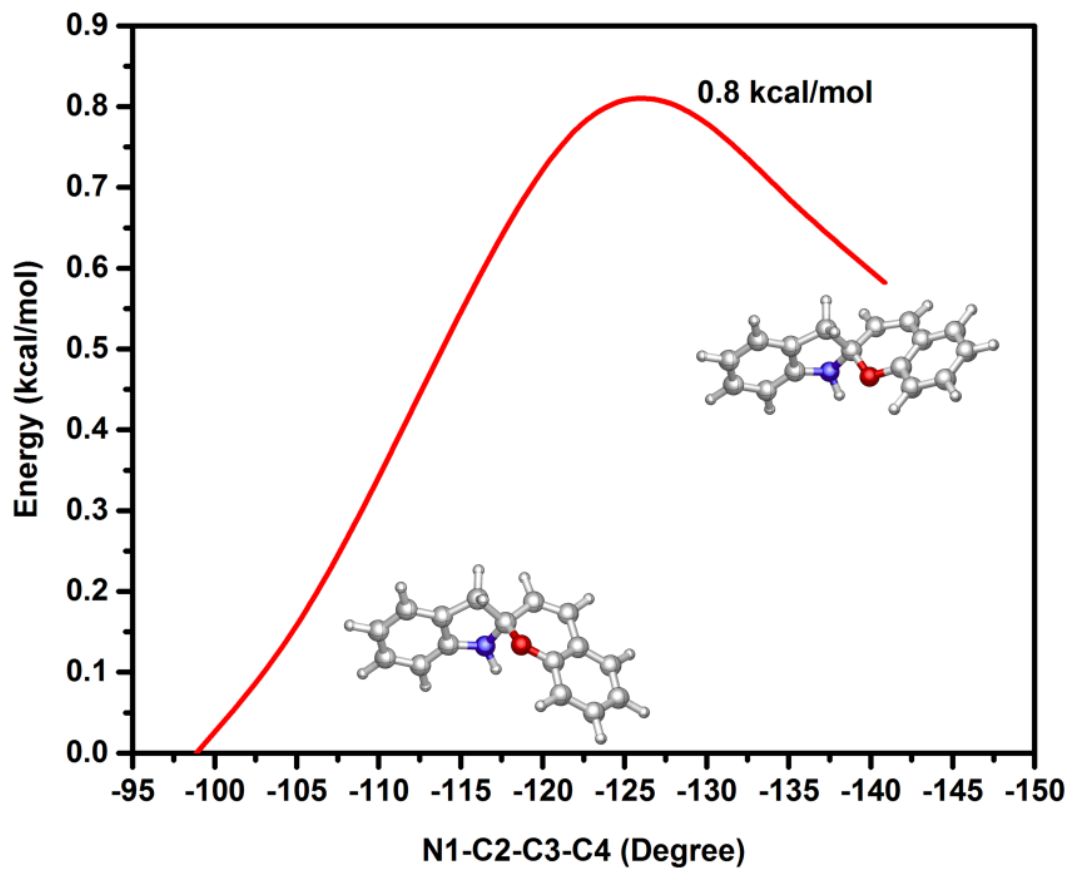

Fig. S5: Energy profile of ground-state conversion from SPc to SPt at the B3LYP+D3/6-31G* level. 


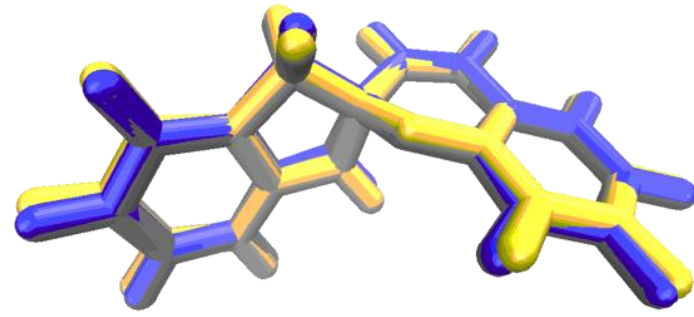

So_SPc

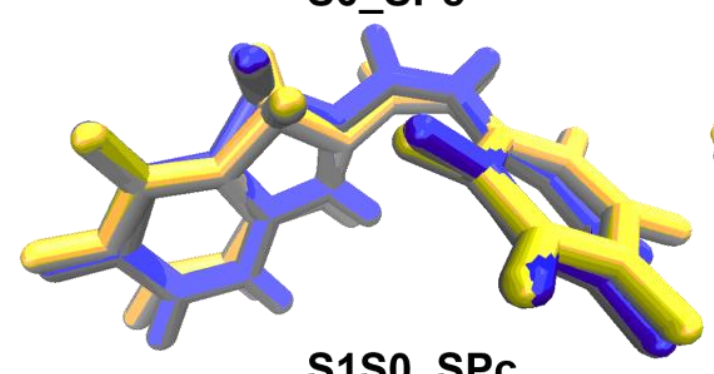

S1S0_SPc

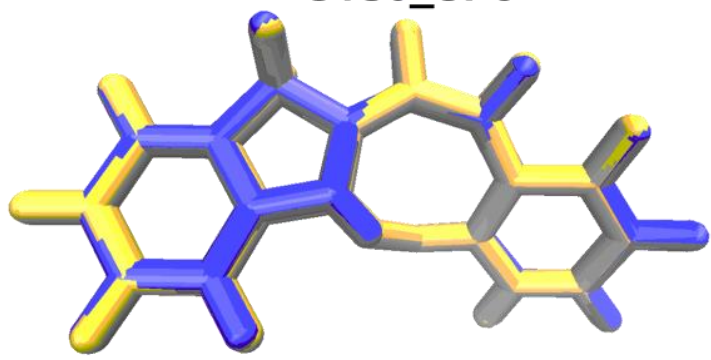

SO_CCC

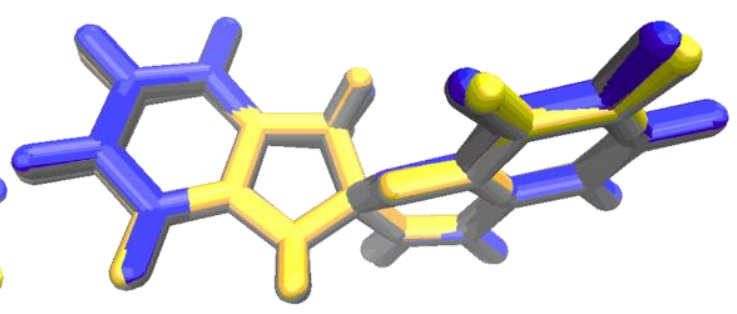

SO_SPt

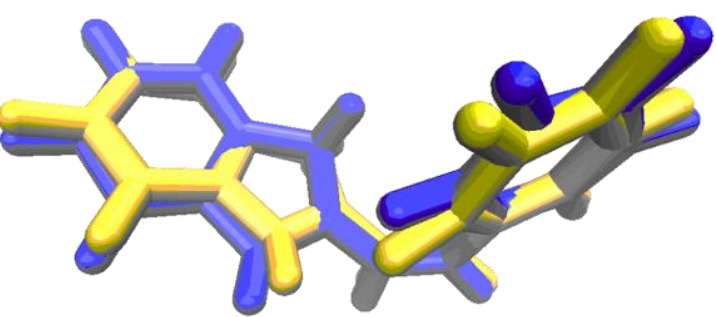

S1S0_SPt

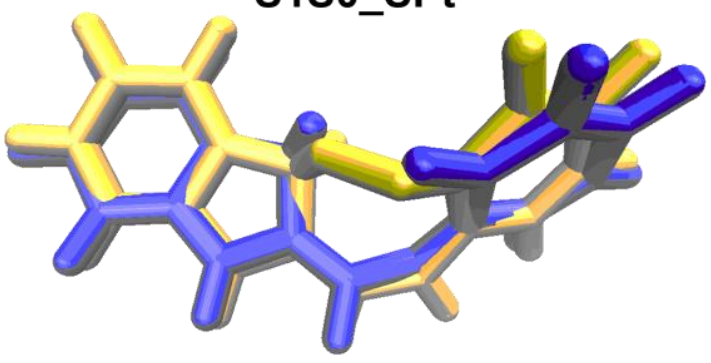

SO_TCC

Fig. S6: Overlap of OM2MRCI (yellow) and CASSCF (blue) optimized structures. The CASSCF structures are taken from the work of Morokuma et al. (ref. 32).
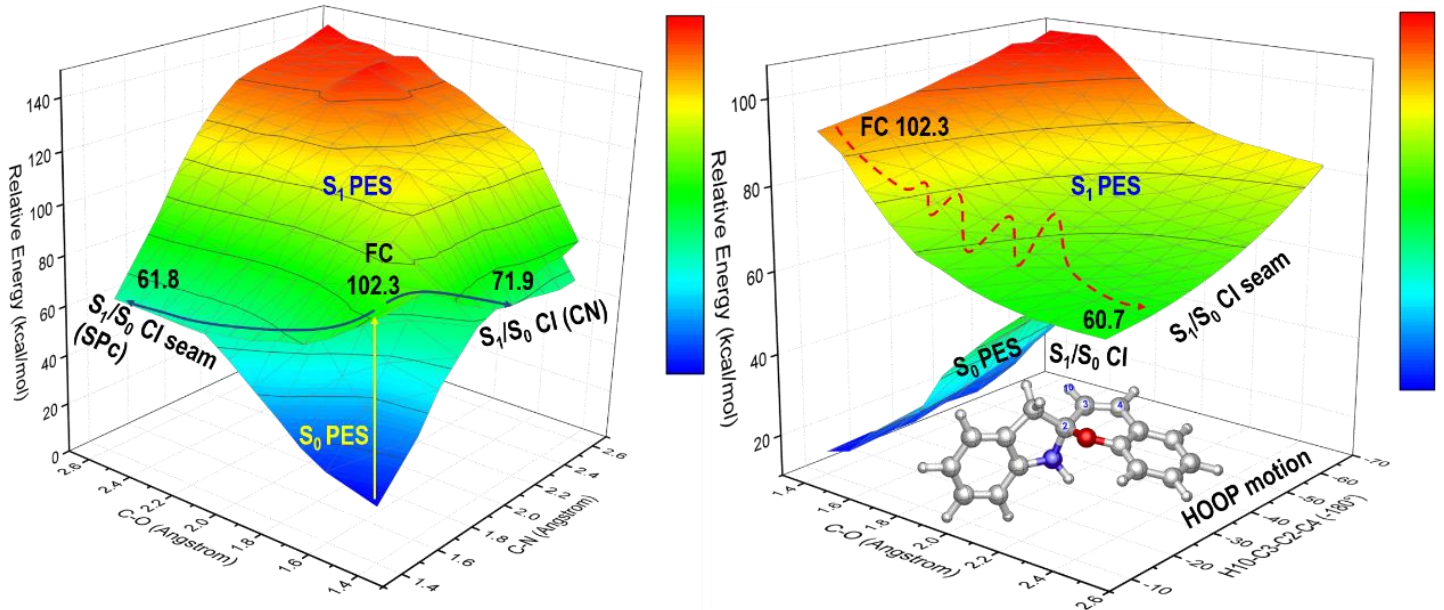

Fig. S7: Calculated two-dimensional potential energy surfaces of both $S_{1}$ and $S_{0}$ states of SPc with respect to (left) the $\mathrm{C}-\mathrm{O}$ and $\mathrm{C}-\mathrm{N}$ bond lengths, and (right) the $\mathrm{C}-\mathrm{O}$ bond length and the $\mathrm{H} 10-\mathrm{C} 3-\mathrm{C} 2-\mathrm{C} 4$ dihedral angle. Minimum-energy reaction paths connecting Franck-Condon points and relevant conical intersections are also shown. Energies are shown in $\mathrm{kcal} / \mathrm{mol}$. 


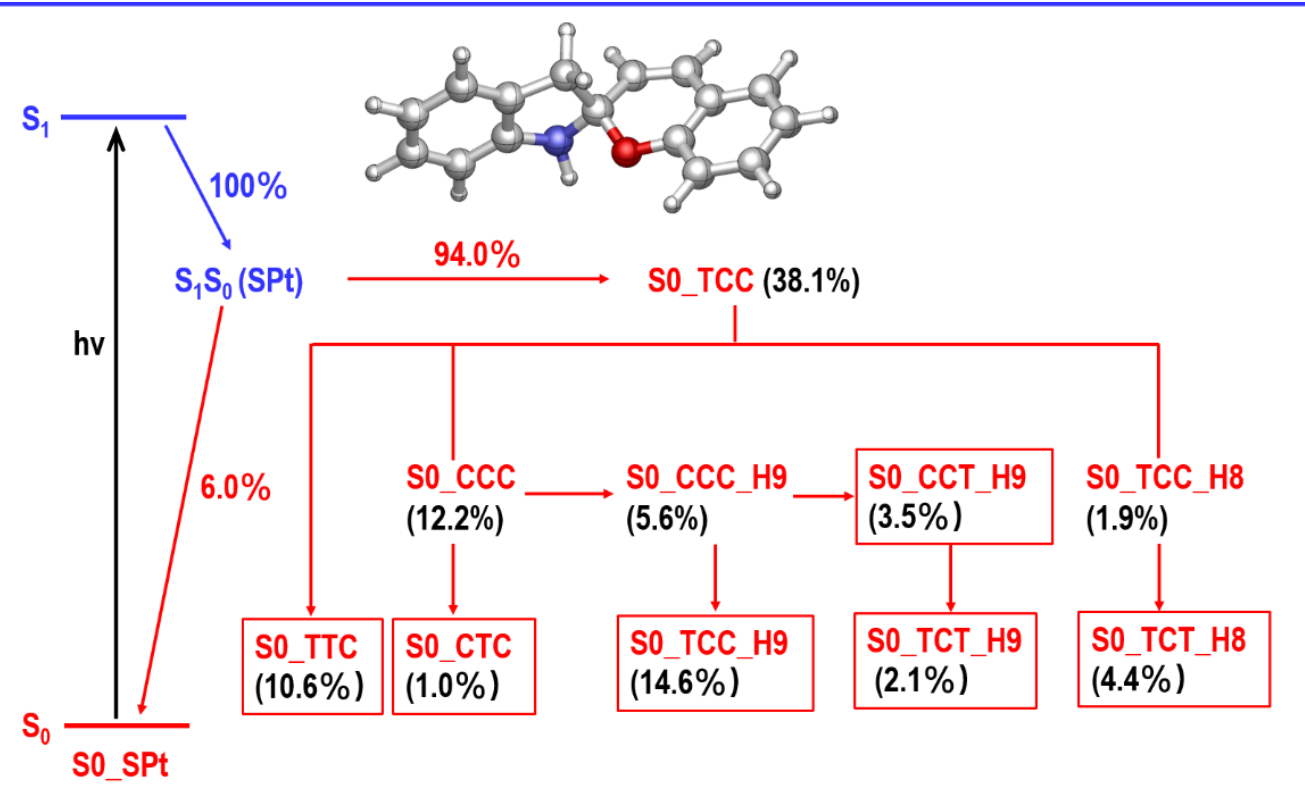

Fig. S8: Suggested photochromic mechanism of SPh starting from SPt conformer. Also shown are the population weights of various intermediates at the end of $500 \mathrm{fs}$ simulation time.
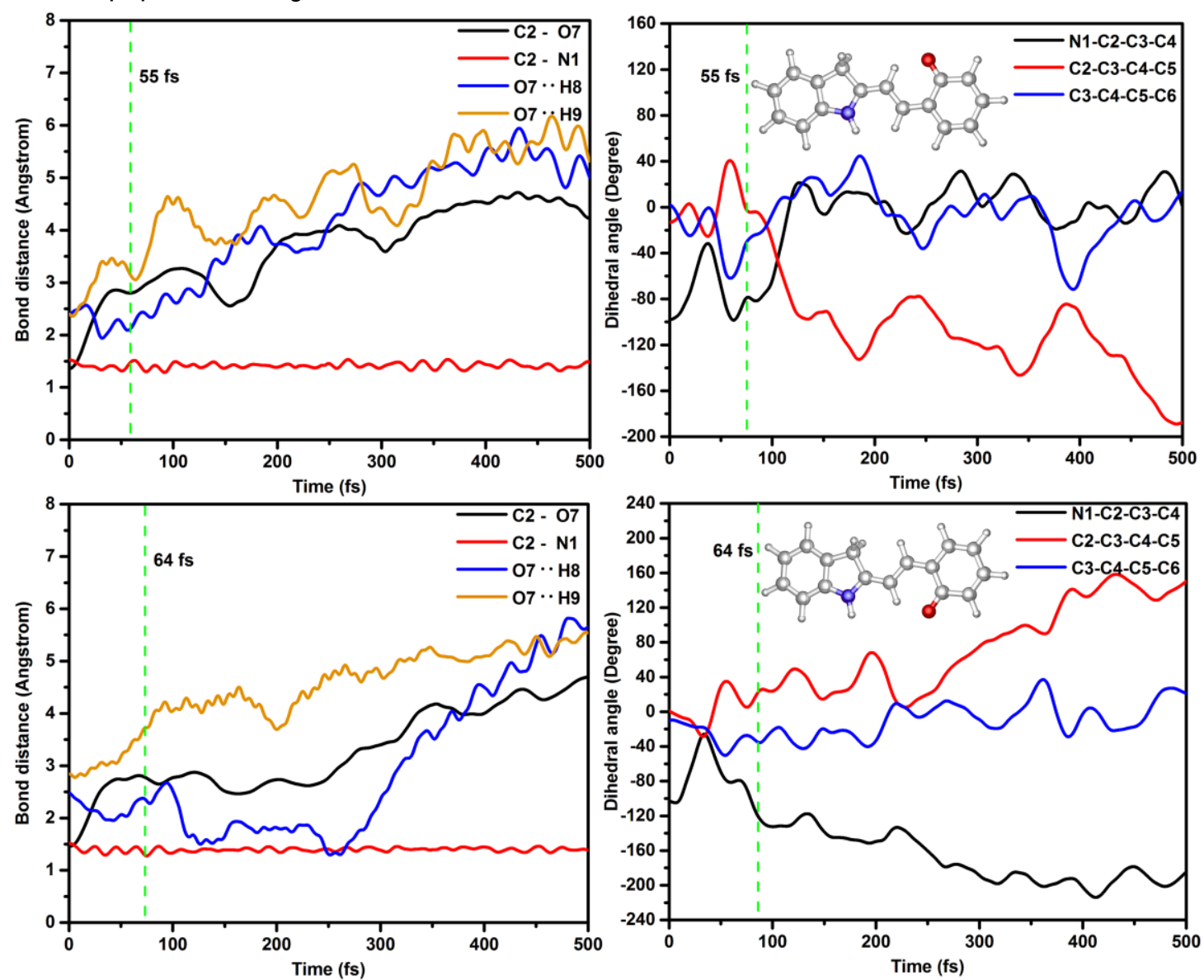

Fig. S9: Time-dependent geometric parameters in two typical trajectories from SPc that finally evolve into S0_CTC (top) and S0_TTC (bottom). Also shown are the $S_{1} \rightarrow S_{0}$ hopping times (see vertical green line). 

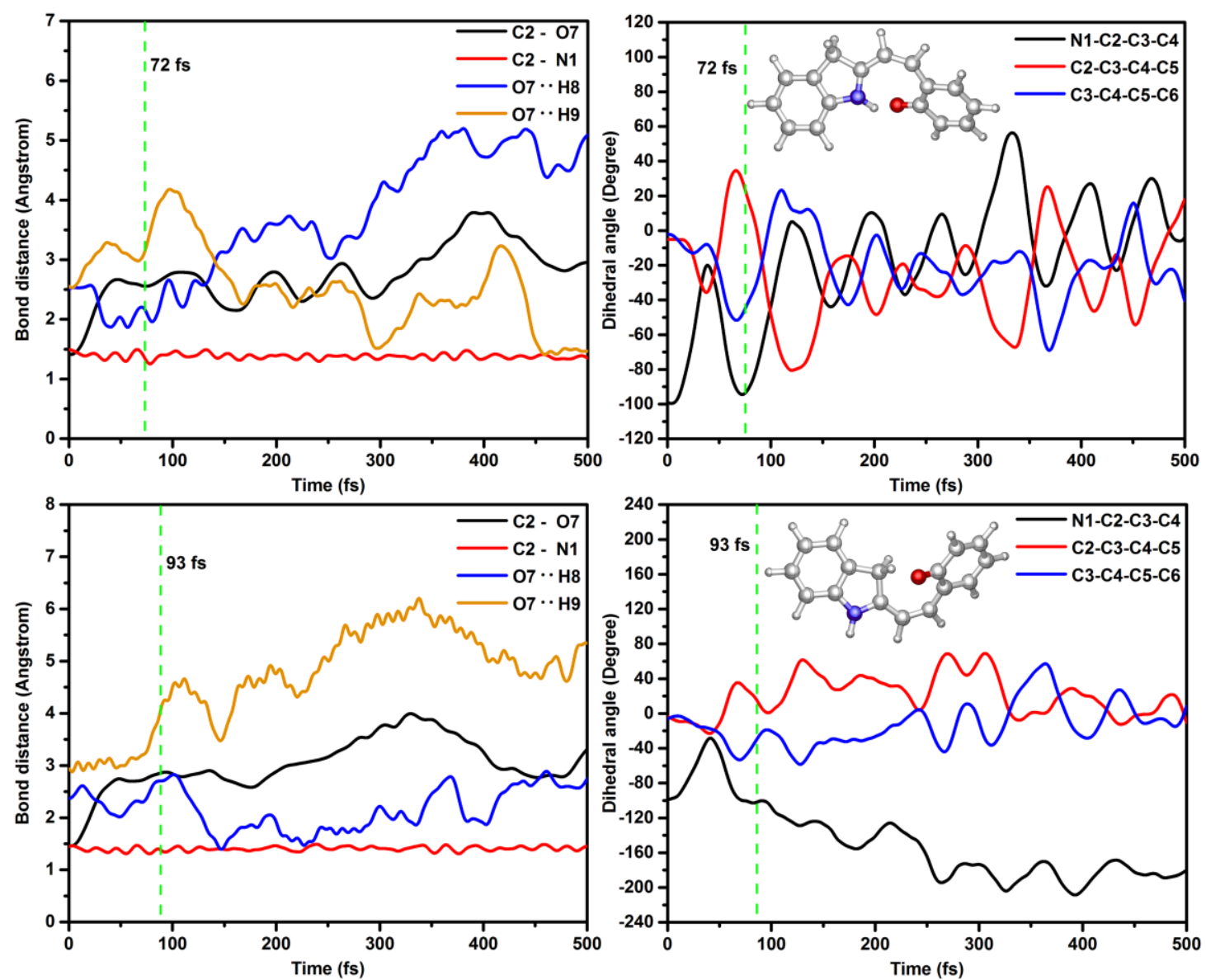

Fig. S10: Time-dependent geometric parameters in two typical trajectories from SPc that finally evolve into S0_CCC (top) and S0_TCC (bottom). Also shown are the $S_{1} \rightarrow S_{0}$ hopping times (see vertical green line).

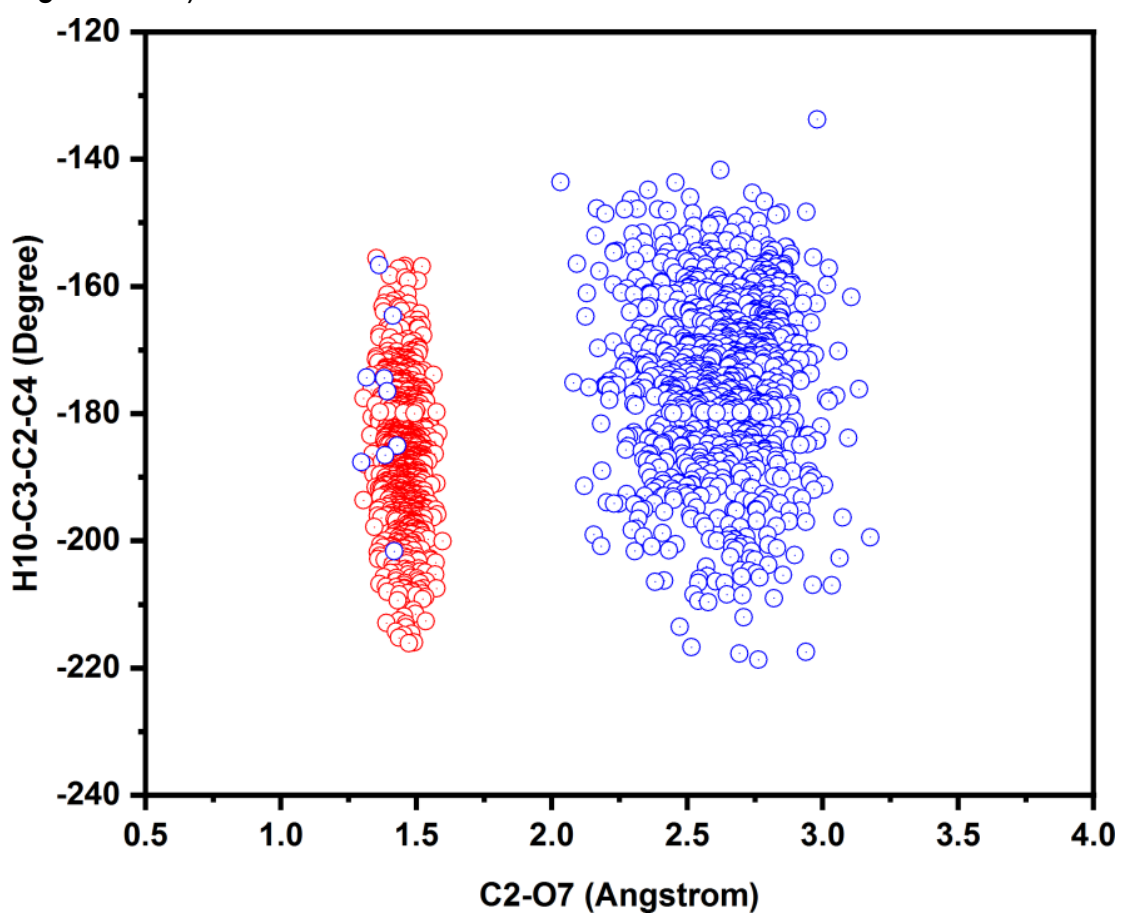

Fig. S11: Geometry parameter distribution of the $\mathrm{C} 2-07$ bond length (in angstrom) and the $\mathrm{H} 10-\mathrm{C} 3-\mathrm{C} 2-\mathrm{C} 4$ dihedral angle (in degree) at all the $\mathrm{S}_{1} \rightarrow \mathrm{S}_{0}$ hopping points for SPc. 


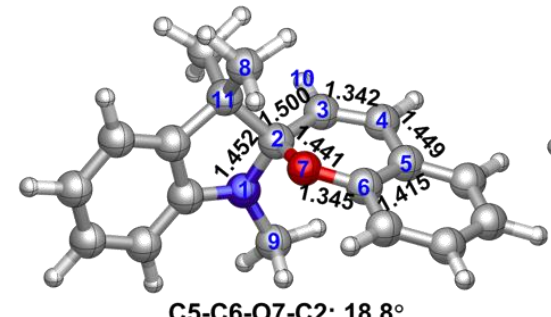

C5-C6-07-C2: 18.8

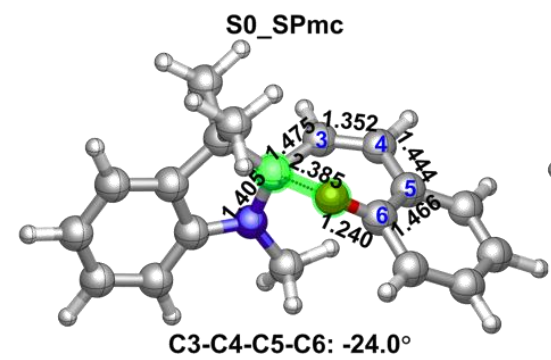

S1S0_SPmc

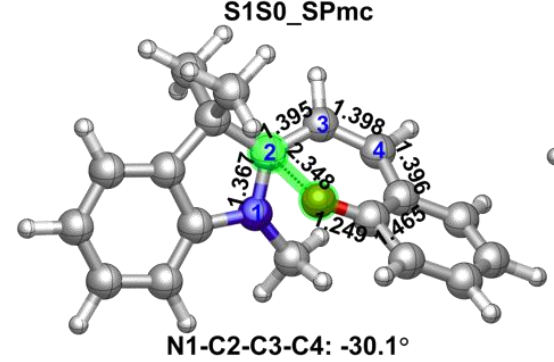

So_CCC

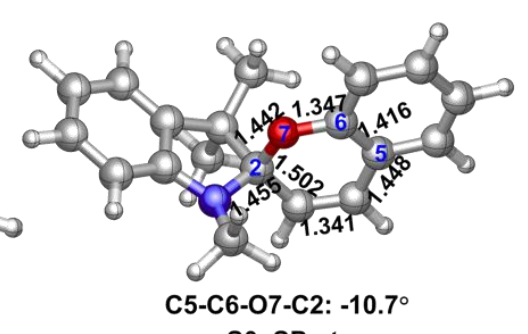

S0_SPmt

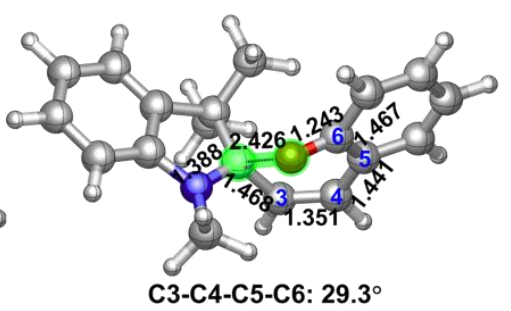

S1S0_SPmt

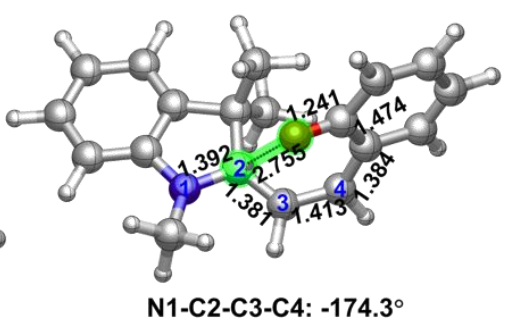

SO_TCC

Fig. S12: OM2/MRCl optimized ground-state equilibrium structures and minimum-energy $S_{1} / S_{0}$ conical intersections of SPmc and SPmt.
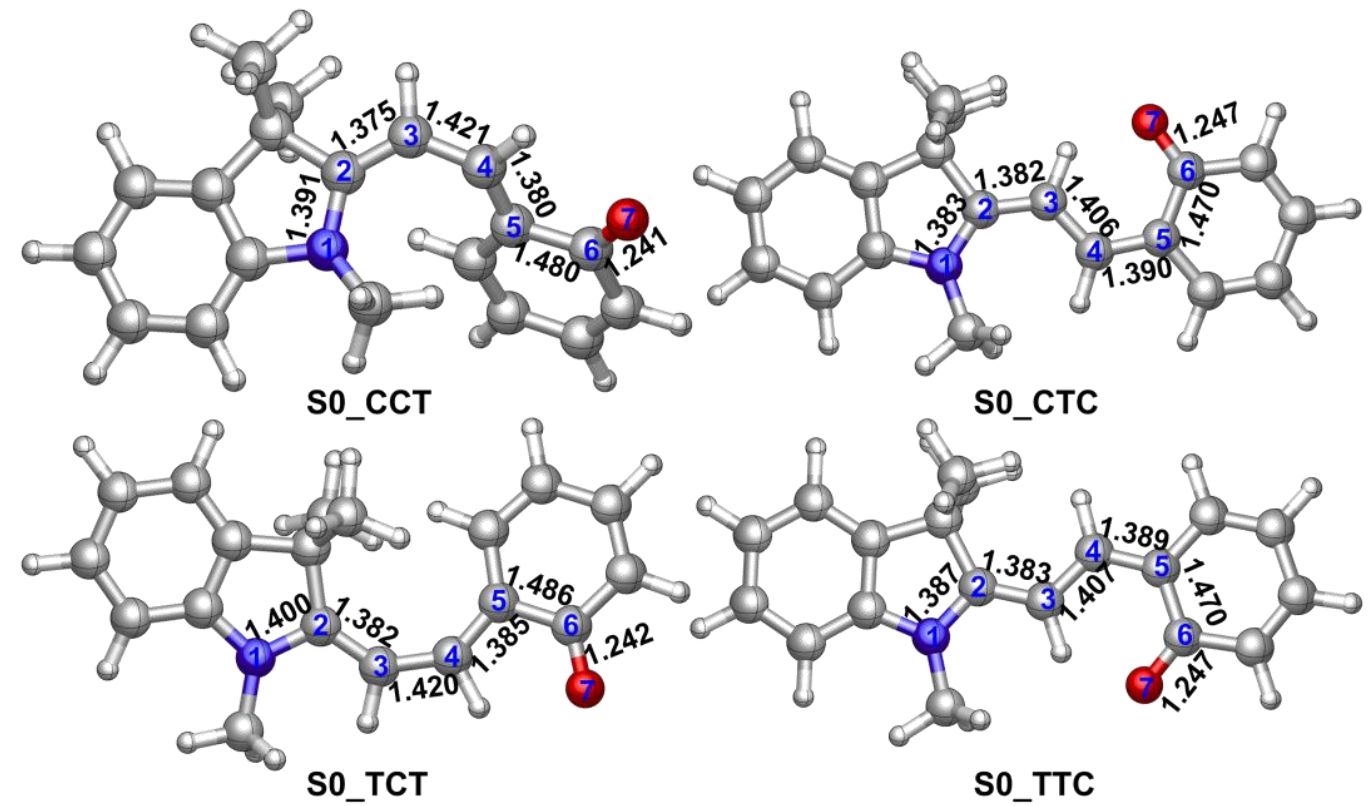

SO_TTC

Fig. S13: OM2MRCI optimized ground-state equilibrium structures of SPm. Also shown are selected bond lengths in angstroms. 

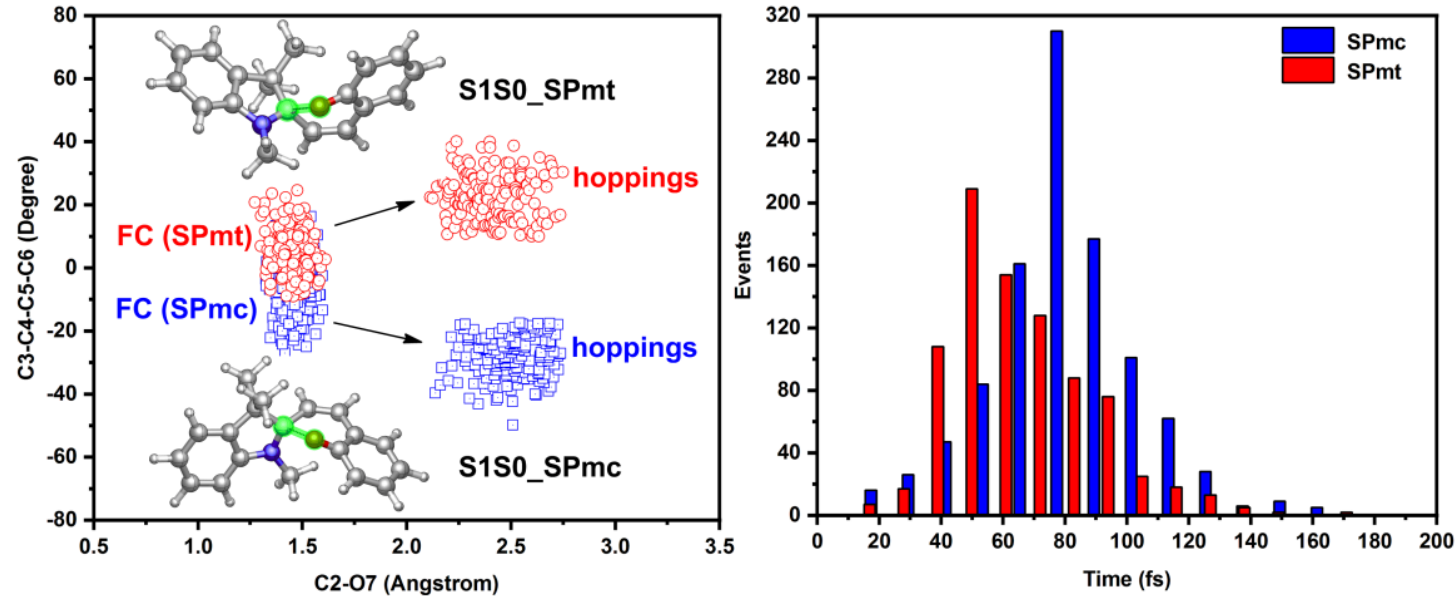

Fig. S14: Distributions of (left) selected geometric parameters and (right) hopping times at all the $\mathrm{S}_{1} \rightarrow \mathrm{S}_{0}$ hopping points.

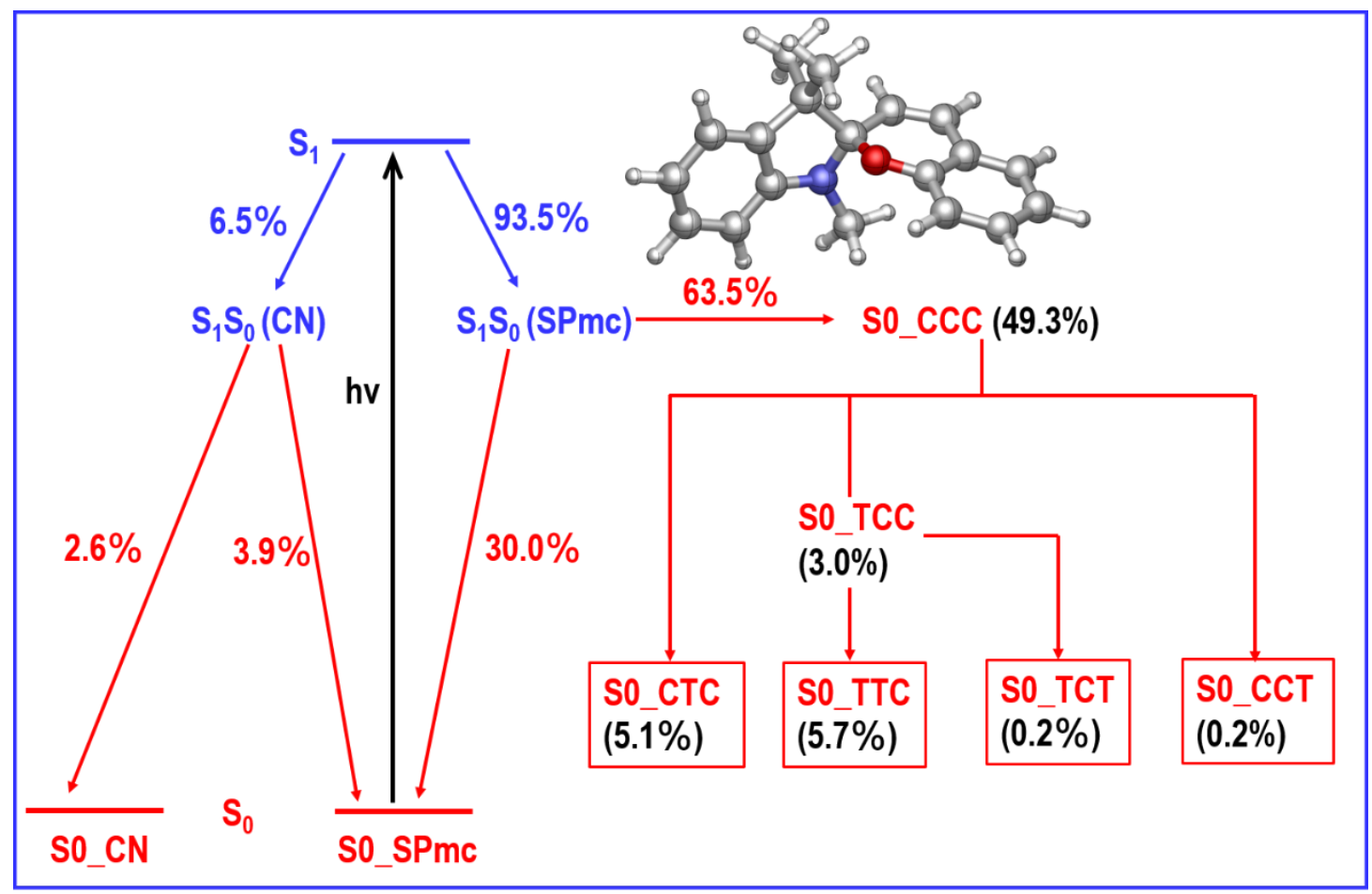

Fig. S15: Suggested photochromic mechanism of SPm starting from SPmc conformer. Also shown are the population weights of various intermediates at the end of $500 \mathrm{fs}$ simulation time. 


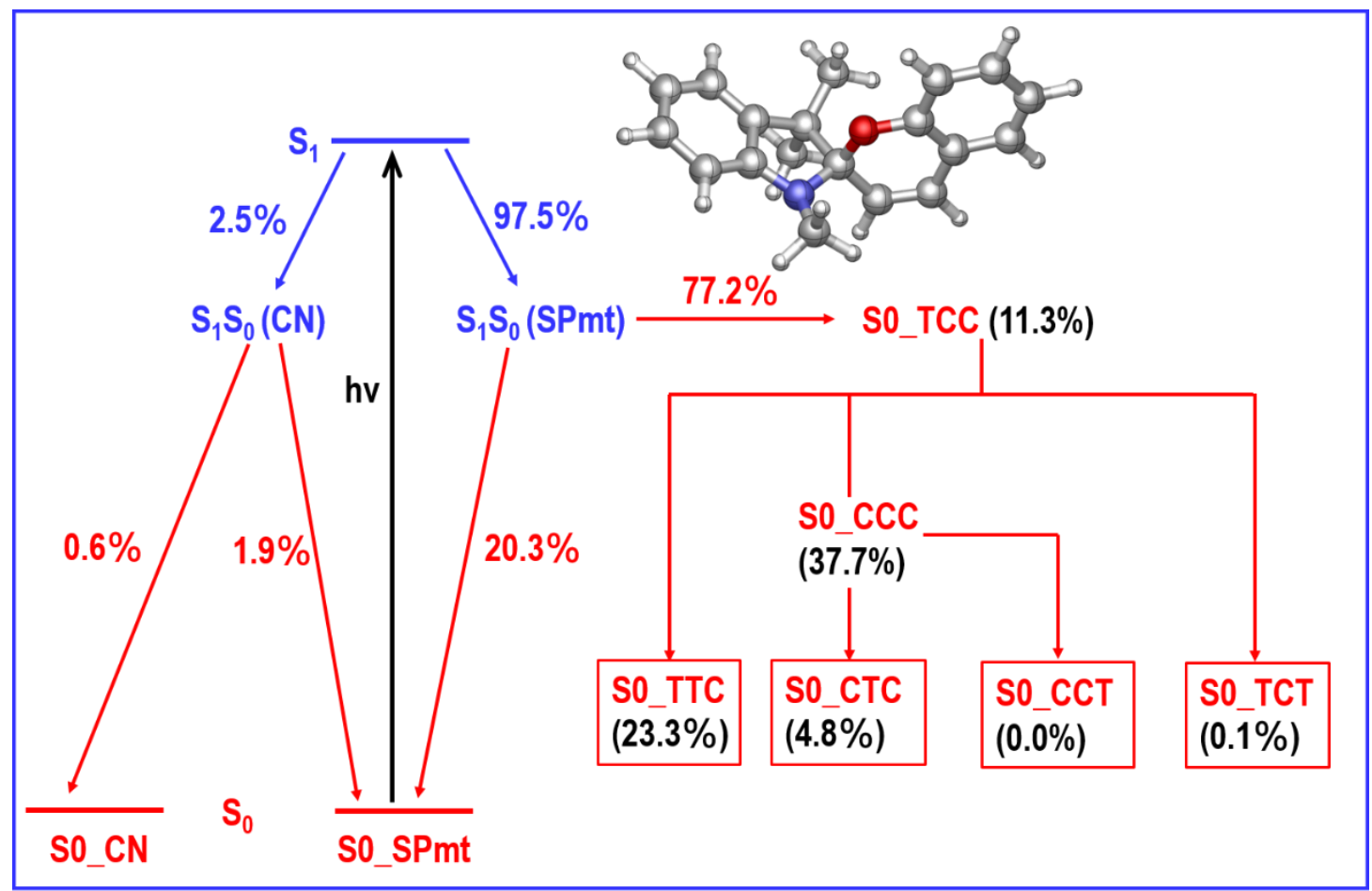

Fig. S16: Suggested photochromic mechanism of SPm starting from SPmt conformer. Also shown are the population weights of various intermediates at the end of $500 \mathrm{fs}$ simulation time.
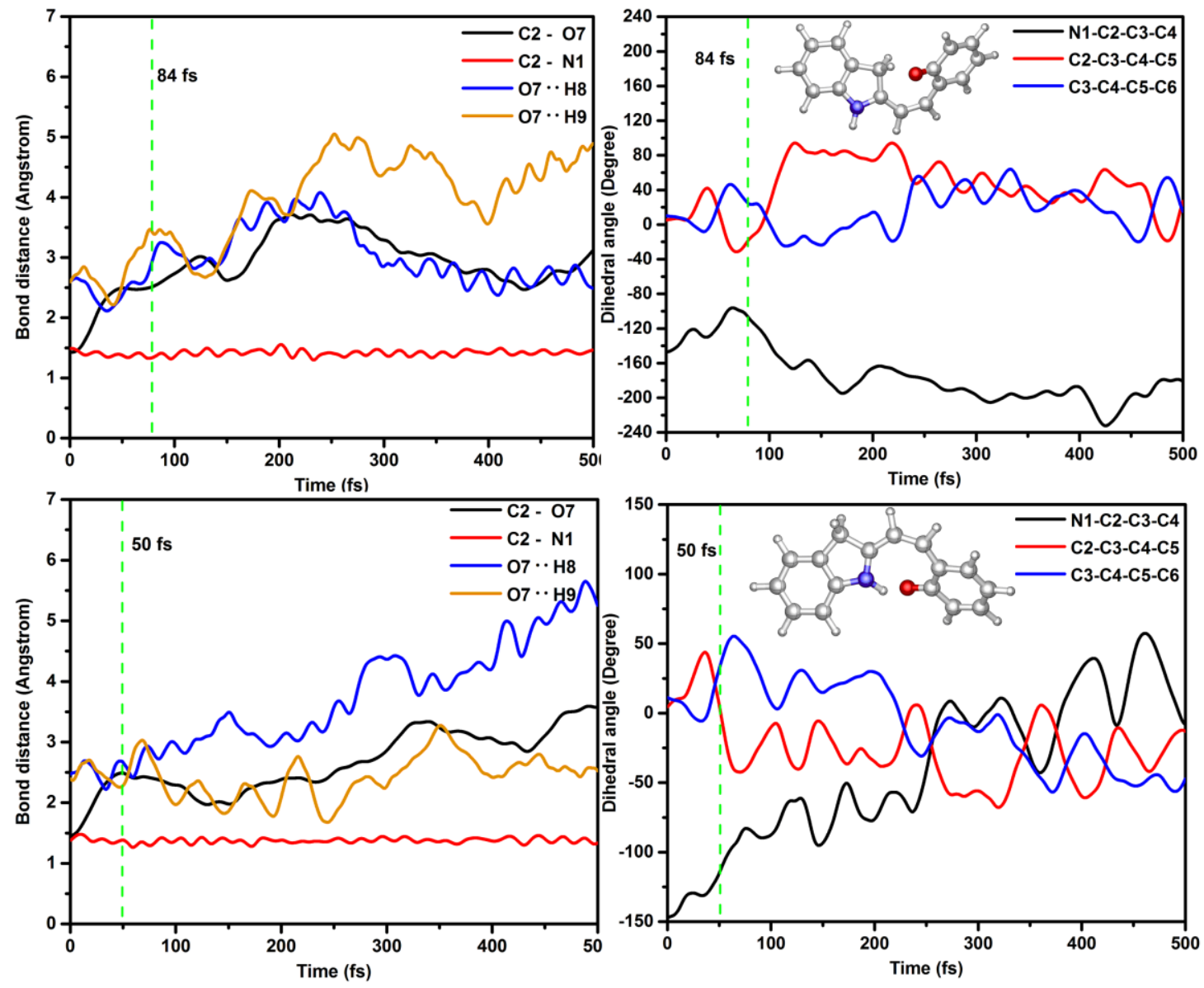
Fig. S17: Time-dependent geometric parameters in two typical trajectories from SPt that finally evolve into S0_TCC (top) and S0_CCC (bottom). Also shown are the $S_{1} \rightarrow S_{0}$ hopping times (see vertical green line).
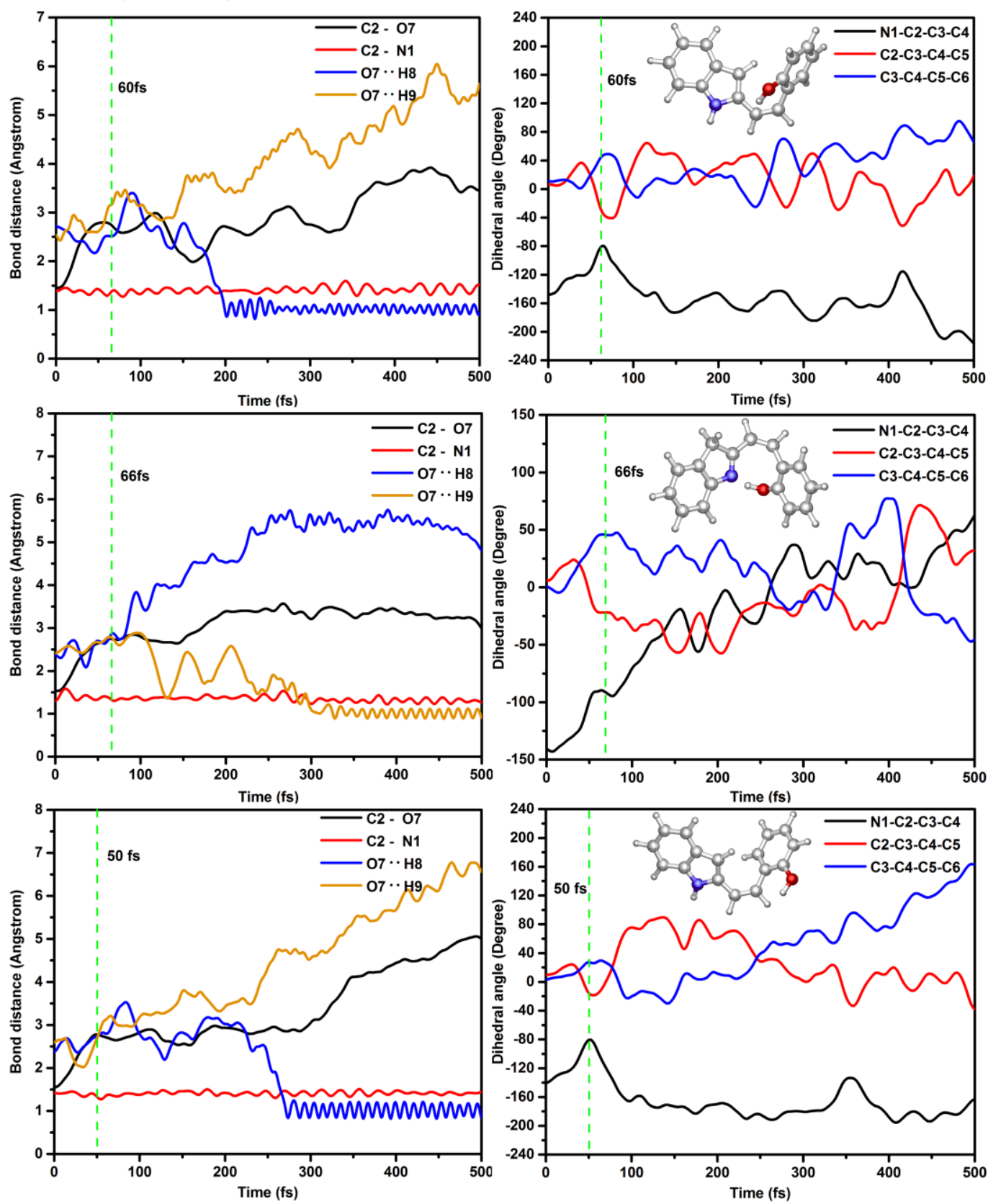

Fig. S18: Time-dependent geometric parameters in three typical trajectories from SPt that finally evolve into SO_TCC_H8 (top), SO_CCC_H9 (middle), and SO_TCT_H8 (bottom). Also shown are the $S_{1} \rightarrow S_{0}$ hopping times (see vertical green line). 

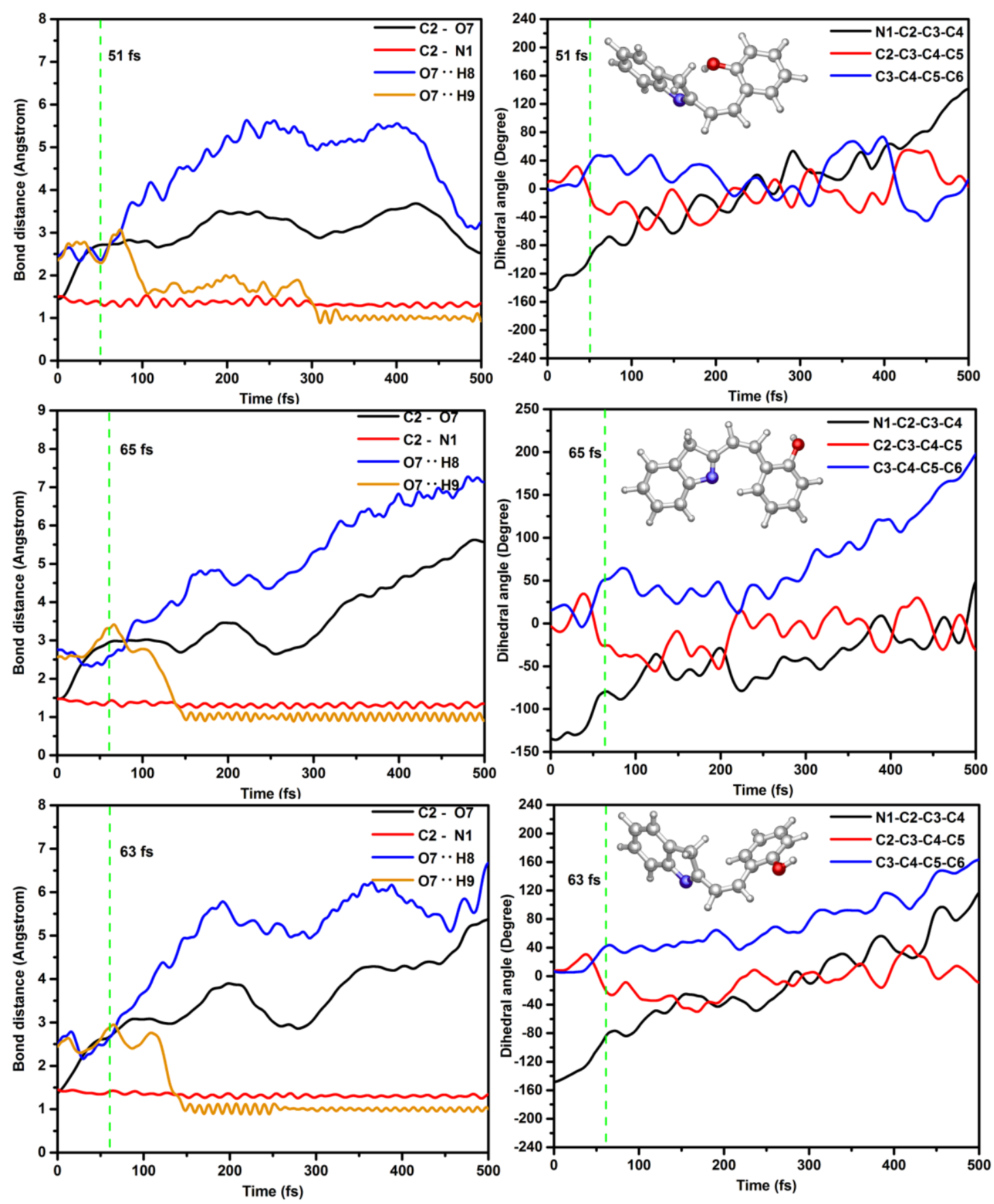

Fig. S19: Time-dependent geometric parameters in two typical trajectories from SPt that finally evolve into SO_TCC_H9 (top), SO_CCT_H9 (middle), and SO_TCT_H9 (bottom). Also shown are the $S_{1} \rightarrow S_{0}$ hopping times (see vertical green line). 

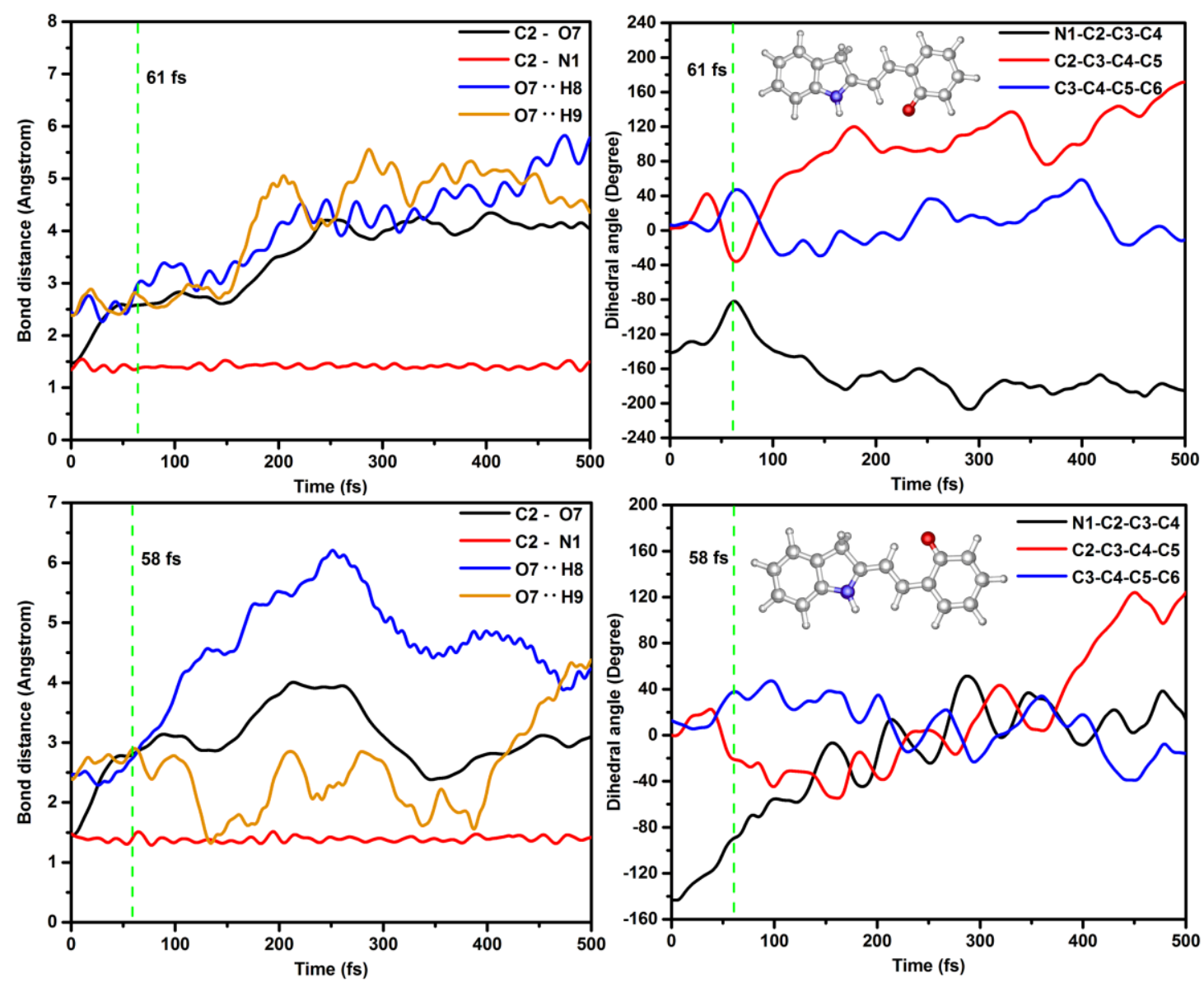

Fig. S20: Time-dependent geometric parameters in two typical trajectories from SPt that finally evolve into S0_TTC (top) and SO_CTC (bottom Also shown are the $S_{1} \rightarrow S_{0}$ hopping times (see vertical green line).

\section{Tables}

Table S1: Computed Relative Energies (in $\mathrm{kcal} / \mathrm{mol}$ ) of the $\mathrm{S}_{0}$ Minima and $\mathrm{S}_{1} / \mathrm{S}_{0}$ Minimum-Energy Conical Intersections (MECls) of SPh. CASSCF and MS-CASPT2//CASSCF Data from Ref. 32 in the Main Text.

\begin{tabular}{lcccccc} 
& \multicolumn{6}{c}{ Relative Energy } \\
\cline { 2 - 7 } Structure & \multicolumn{2}{c}{ OM2/MRCI } & \multicolumn{2}{c}{ CASSCF } & \multicolumn{2}{c}{ MS-CASPT2 } \\
\cline { 2 - 7 } & $\mathbf{S}_{0}$ & $\mathbf{S}_{1}$ & $\mathbf{S}_{0}$ & $\mathbf{S}_{1}$ & $\mathbf{S}_{0}$ & $\mathbf{S}_{1}$ \\
S0_SPc & 1.2 & $/$ & 0.1 & $/$ & 0.0 & $/$ \\
S0_SPt & 0.0 & $/$ & 0.0 & $/$ & 0.3 & $/$ \\
S0_CCC & 18.3 & $/$ & 25.5 & $/$ & 25.1 & $/$ \\
S0_TCC & 19.1 & $/$ & 26.1 & $/$ & 34.1 & $/$ \\
S1S0_SPc & 60.5 & 60.5 & 66.5 & 67.8 & 45.2 & 68.8 \\
S1S0_SPt & 57.8 & 57.8 & 72.9 & 73.2 & 42.4 & 69.1
\end{tabular}


Table S2: computed energies (eV/nm), oscillator strengths, and electronic configurations of SPc.

\begin{tabular}{|c|c|c|c|}
\hline & MO (weight \%) & Energy (eV/nm) & Osc. \\
\hline $\mathrm{S}_{0} \rightarrow \mathrm{S}_{1}$ & HOMO $\rightarrow$ LUMO (84) & $4.44 / 279$ & 0.18 \\
\hline $\mathrm{S}_{0} \rightarrow \mathrm{S}_{2}$ & HOMO-1 $\rightarrow$ LUMO+1 (41) & $5.19 / 239$ & 0.04 \\
\hline
\end{tabular}

Table S3: computed energies (eV/nm), oscillator strengths, and electronic configurations of SPt.

\begin{tabular}{|c|c|c|c|}
\hline & MO (weight \%) & Energy (eV/nm) & Osc. \\
\hline $\mathrm{S}_{0} \rightarrow \mathrm{S}_{1}$ & HOMO $\rightarrow$ LUMO (84) & $4.39 / 282$ & 0.24 \\
\hline $\mathrm{S}_{0} \rightarrow \mathrm{S}_{2}$ & HOMO-2 $\rightarrow$ LUMO (32) & $5.26 / 236$ & 0.00 \\
\hline
\end{tabular}

Table S4: computed energies (eV/nm), oscillator strengths, and electronic configurations of SPmc.

\begin{tabular}{|c|c|c|c|}
\hline & MO (weight \%) & Energy (eV/nm) & Osc. \\
\hline $\mathrm{S}_{0} \rightarrow \mathrm{S}_{1}$ & HOMO $\rightarrow$ LUMO (82) & $4.34 / 286$ & 0.28 \\
\hline $\mathrm{S}_{0} \rightarrow \mathrm{S}_{2}$ & HOMO-1 $\rightarrow$ LUMO+1 (36) & $5.14 / 241$ & 0.05 \\
\hline
\end{tabular}

Table S5: computed energies (eV/nm), oscillator strengths, and electronic configurations of SPmt.

\begin{tabular}{|c|c|c|c|}
\hline & MO (weight \%) & Energy (eV/nm) & Osc. \\
\hline $\mathrm{S}_{0} \rightarrow \mathrm{S}_{1}$ & HOMO $\rightarrow$ LUMO (83) & $4.32 / 287$ & 0.27 \\
\hline $\mathrm{S}_{0} \rightarrow \mathrm{S}_{2}$ & HOMO-1 $\rightarrow$ LUMO+1 (56) & $5.17 / 240$ & 0.03 \\
\hline
\end{tabular}




\section{Cartesian coordinates}

31

SO_SPC

$\begin{array}{lrrr}\text { C } & -0.0161837200 & 0.1615762670 & -0.0240460770 \\ \mathrm{C} & -0.0538162150 & 0.1916950460 & 1.5311484630 \\ \mathrm{~N} & 1.3614112660 & 0.3683673290 & -0.4146041330 \\ \mathrm{C} & -0.5902115770 & -1.0753857020 & -0.6605205740 \\ \mathrm{C} & 1.3217666670 & 0.6689814570 & 1.8817503600 \\ \mathrm{C} & 2.1001214820 & 0.7813165910 & 0.6956407960 \\ \mathrm{C} & -1.3098270260 & -1.0073663710 & -1.7934909260 \\ \mathrm{C} & 1.8726220380 & 0.9867118200 & 3.1126896250 \\ \mathrm{C} & 3.4294597790 & 1.2110859180 & 0.7386301010 \\ \mathrm{C} & -1.5291902730 & 0.2939063150 & -2.4039716260 \\ \mathrm{C} & 3.2041085220 & 1.4181032370 & 3.1556767820 \\ \mathrm{C} & 3.9640734840 & 1.5254314300 & 1.9894612010 \\ \mathrm{C} & -1.2237677570 & 1.4404982370 & -1.6360943670 \\ \mathrm{C} & -2.0319733200 & 0.4653421840 & -3.6996544080 \\ \mathrm{O} & -0.7482980870 & 1.3366559470 & -0.3737276100 \\ \mathrm{C} & -1.4071991160 & 2.7387220420 & -2.1341163290 \\ \mathrm{C} & -2.2172790220 & 1.7476037790 & -4.2045264240 \\ \mathrm{C} & -1.9129459260 & 2.8730833560 & -3.4234101200 \\ \mathrm{H} & -2.0816831560 & 3.8748828910 & -3.8322919450 \\ \mathrm{H} & -0.8307815440 & 0.8925615370 & 1.8786306300 \\ \mathrm{H} & -0.2421559540 & -0.8228281230 & 1.9297593170 \\ \mathrm{H} & 1.5837937390 & 0.7118759460 & -1.3377295590 \\ \mathrm{H} & -0.3604846040 & -2.0214163520 & -0.1683062220 \\ \mathrm{H} & -1.7528382800 & -1.8882242960 & -2.2670450350 \\ \mathrm{H} & 1.2813005100 & 0.9058855950 & 4.0302744820 \\ \mathrm{H} & 4.0212733420 & 1.2947105730 & -0.1742268780 \\ \mathrm{H} & 3.6544546380 & 1.6770328640 & 4.1196144380 \\ \mathrm{H} & 5.0053823910 & 1.8646985890 & 2.0554382250 \\ \mathrm{H} & -2.2765987510 & -0.4153444440 & -4.3054283510 \\ \mathrm{H} & -1.1607586950 & 3.5988031370 & -1.5153062890 \\ \mathrm{H} & -2.6114704460 & 1.8848623520 & -5.2157609710\end{array}$

31

SO_SPt

$\begin{array}{lrrr}C & -0.0214338020 & 0.1931034130 & -0.0674331450 \\ \mathrm{C} & -0.0981735080 & 0.3336087780 & 1.4915464900 \\ \mathrm{~N} & 1.3778292730 & 0.2686266940 & -0.4351173960 \\ \mathrm{C} & -0.6886254150 & -1.0632229490 & -0.5518406380 \\ \mathrm{C} & 1.3004332300 & 0.7301464170 & 1.8472228640 \\ \mathrm{C} & 2.1104858480 & 0.7007729050 & 0.6794185110\end{array}$




$\begin{array}{lrrr}\text { C } & -1.9387365510 & -1.0376998510 & -1.0548394120 \\ \text { C } & 1.8464716140 & 1.0894701830 & 3.0699364260 \\ \text { C } & 3.4681727560 & 1.0281456930 & 0.7317236670 \\ \text { C } & -2.6352771800 & 0.2387537000 & -1.1020094840 \\ \text { C } & 3.2067103220 & 1.4171192320 & 3.1214991140 \\ \text { C } & 3.9991655970 & 1.3845989030 & 1.9723616450 \\ \text { C } & -1.8852179250 & 1.4128288230 & -0.8923762150 \\ \text { C } & -4.0099090150 & 0.3552480650 & -1.3569348080 \\ \text { O } & -0.5479921180 & 1.3724060520 & -0.6471708490 \\ \text { C } & -2.4563887630 & 2.6895668000 & -0.9432300820 \\ \text { C } & -4.5896766130 & 1.6179961830 & -1.4271976960 \\ \text { C } & -3.8181615070 & 2.7731855680 & -1.2387772740 \\ \text { H } & -4.2833896650 & 3.7594477560 & -1.3445427800 \\ \text { H } & -0.8289084020 & 1.1115407980 & 1.7721537840 \\ \text { H } & -0.3699051050 & -0.6315755500 & 1.9561947600 \\ \text { H } & 1.5943970400 & 0.6865395380 & -1.3362176500 \\ \text { H } & -0.1104919120 & -1.9800371190 & -0.4488278800 \\ \text { H } & -2.4357324820 & -1.9268021900 & -1.4514394390 \\ \text { H } & 1.2299116120 & 1.1158972770 & 3.9738173340 \\ \text { H } & 4.0845443380 & 1.0023521580 & -0.1686098580 \\ \text { H } & 3.6562862440 & 1.6983379030 & 4.0799318930 \\ \text { H } & 5.0624522030 & 1.6435714490 & 2.0443307480 \\ \text { H } & -4.6106784660 & -0.5488369270 & -1.5059152570 \\ \text { H } & -1.8471171170 & 3.5730556560 & -0.7723003800 \\ \text { H } & -5.6580734070 & 1.7153224720 & -1.6440313610\end{array}$

31

S1S0_SPC

$\begin{array}{lrrr}C & 0.2669803810 & -0.1597809250 & -0.0220879620 \\ \mathrm{C} & 0.0517131050 & 0.1422133470 & 1.4473300570 \\ \mathrm{~N} & 1.5946885730 & 0.0362907770 & -0.3479257170 \\ \mathrm{C} & -0.5045286320 & -1.1646737020 & -0.7522887010 \\ \mathrm{C} & 1.4026181100 & 0.6504210640 & 1.8624231770 \\ \mathrm{C} & 2.2832261260 & 0.5812548020 & 0.7432922280 \\ \mathrm{C} & -1.3510774750 & -0.8896258850 & -1.7707205510 \\ \mathrm{C} & 1.8672861430 & 1.1503761060 & 3.0684210210 \\ \mathrm{C} & 3.6111355240 & 1.0088966290 & 0.8141891410 \\ \mathrm{C} & -1.6267012270 & 0.4247555000 & -2.2846033850 \\ \mathrm{C} & 3.1991221450 & 1.5750937560 & 3.1482371470 \\ \mathrm{C} & 4.0503238940 & 1.5053391660 & 2.0429403190 \\ \mathrm{C} & -1.6030314210 & 1.6258413580 & -1.4450974080 \\ \mathrm{C} & -1.9539868440 & 0.5518114200 & -3.6430745090 \\ \mathrm{O} & -1.5150488200 & 1.5682623770 & -0.2045264990 \\ \mathrm{C} & -1.7875005240 & 2.9073464730 & -2.1086835720\end{array}$




$\begin{array}{lrrr}\mathrm{C} & -2.1584278960 & 1.7970688860 & -4.2190667780 \\ \mathrm{C} & -2.0550879290 & 2.9771006380 & -3.4485075820 \\ \mathrm{H} & -2.2035974550 & 3.9445258800 & -3.9427202710 \\ \mathrm{H} & -0.7276353590 & 0.9151494910 & 1.5381060820 \\ \mathrm{H} & -0.2360258240 & -0.7707011960 & 2.0146012910 \\ \mathrm{H} & 1.9854061270 & -0.0779616440 & -1.2693630030 \\ \mathrm{H} & -0.3098991440 & -2.2124540490 & -0.4666316870 \\ \mathrm{H} & -1.8377951380 & -1.7274611970 & -2.2987656380 \\ \mathrm{H} & 1.2068972970 & 1.2154170930 & 3.9381963420 \\ \mathrm{H} & 4.2722780180 & 0.9547184290 & -0.0527799090 \\ \mathrm{H} & 3.5796124320 & 1.9770447500 & 4.0936483520 \\ \mathrm{H} & 5.0876262780 & 1.8484944400 & 2.1399347850 \\ \mathrm{H} & -2.0302893490 & -0.3487069760 & -4.2677679210 \\ \mathrm{H} & -1.7169042710 & 3.8028880010 & -1.4869715380 \\ \mathrm{H} & -2.3990689830 & 1.8748810400 & -5.2852791350\end{array}$

31

S1S0_SPt

$\begin{array}{lrrr}\text { C } & 0.0290841070 & -0.3276351190 & 0.2036241230 \\ \text { C } & 0.0297990230 & -0.3075495830 & 1.7184545090 \\ \text { N } & 1.2045531060 & 0.1794113550 & -0.2895510590 \\ \text { C } & -0.7775215630 & -1.2980921570 & -0.5299124030 \\ \text { C } & 1.2913786190 & 0.4526749510 & 2.0073782360 \\ \text { C } & 1.9396655750 & 0.7379292460 & 0.7708972130 \\ \text { C } & -1.9472735670 & -0.9892869190 & -1.1305403620 \\ \text { C } & 1.8602906100 & 0.8630489310 & 3.2019080530 \\ \text { C } & 3.1489821760 & 1.4369159110 & 0.7198679880 \\ \text { C } & -2.4752496680 & 0.3461012360 & -1.2168829110 \\ \text { C } & 3.0696847260 & 1.5691852760 & 3.1534140390 \\ \text { C } & 3.6978889610 & 1.8471051610 & 1.9347558920 \\ \text { C } & -1.6027208600 & 1.4928738570 & -1.4735340760 \\ \text { C } & -3.8587405370 & 0.5490654910 & -1.1076664970 \\ \text { O } & -0.4100024090 & 1.3552325550 & -1.8112809310 \\ \text { C } & -2.2099365300 & 2.8129474270 & -1.4356969550 \\ \text { C } & -4.3934787160 & 1.8291547020 & -1.1296915460 \\ \text { C } & -3.5603437930 & 2.9598230880 & -1.2737660890 \\ \text { H } & -4.0182655810 & 3.9562036370 & -1.2686478450 \\ \text { H } & -0.8695976090 & 0.2282460290 & 2.0866861740 \\ \text { H } & 0.0562757220 & -1.3321769590 & 2.1484914690 \\ \text { H } & 1.2408068630 & 0.5437750210 & -1.2402606600 \\ \text { H } & -0.4018366080 & -2.3352507610 & -0.5312949020 \\ \text { H } & -2.5785979660 & -1.7930064570 & -1.5390798500 \\ \text { H } & 1.3756476080 & 0.6485853240 & 4.1595232940 \\ \text { H } & 3.6353371080 & 1.6504236430 & -0.2335553610\end{array}$




$\begin{array}{lrrr}H & 3.5280539180 & 1.9151960160 & 4.0859487190 \\ \mathrm{H} & 4.6434175220 & 2.4032276750 & 1.9340152600 \\ \mathrm{H} & -4.5239144370 & -0.3135871820 & -0.9770694040 \\ \mathrm{H} & -1.5465142800 & 3.6699865770 & -1.5665571340 \\ \mathrm{H} & -5.4738987200 & 1.9729421920 & -1.0186532860\end{array}$

31

SO_CCC (SPh)

$\begin{array}{lrrr}\mathrm{C} & 0.0599911000 & 0.0721835000 & -0.0644283000 \\ \mathrm{C} & 0.0148019000 & -0.2135483000 & 1.4400303000 \\ \mathrm{~N} & 1.3293518000 & 0.2485351000 & -0.4827021000 \\ \mathrm{C} & -1.1396882000 & 0.2444815000 & -0.7655463000 \\ \mathrm{C} & 1.4685802000 & -0.2039134000 & 1.7954430000 \\ \mathrm{C} & 2.2055460000 & 0.0786041000 & 0.6120227000 \\ \mathrm{C} & -1.3855346000 & 0.5408134000 & -2.1054298000 \\ \mathrm{C} & 2.1174394000 & -0.3976352000 & 3.0042777000 \\ \mathrm{C} & 3.5965945000 & 0.1986539000 & 0.6327823000 \\ \mathrm{C} & -0.6588777000 & 0.4307024000 & -3.2977449000 \\ \mathrm{C} & 3.5123618000 & -0.2887831000 & 3.0212614000 \\ \mathrm{C} & 4.2327734000 & 0.0143636000 & 1.8629214000 \\ \mathrm{C} & 0.5492587000 & -0.3416251000 & -3.5167584000 \\ \mathrm{C} & -1.2797704000 & 1.0326509000 & -4.4452199000 \\ \mathrm{O} & 1.1776507000 & -0.9185060000 & -2.5822209000 \\ \mathrm{C} & 1.0095130000 & -0.5323332000 & -4.8764503000 \\ \mathrm{C} & -0.7900943000 & 0.8527104000 & -5.7111901000 \\ \mathrm{C} & 0.3428602000 & 0.0247150000 & -5.9283022000 \\ \mathrm{H} & 0.6645774000 & -0.1604590000 & -6.9613384000 \\ \mathrm{H} & -0.5356073000 & 0.5739887000 & 1.9816677000 \\ \mathrm{H} & -0.4451293000 & -1.2023418000 & 1.6073034000 \\ \mathrm{H} & 1.5736054000 & -0.0812349000 & -1.4832898000 \\ \mathrm{H} & -2.0372833000 & 0.1914383000 & -0.1282443000 \\ \mathrm{H} & -2.4275844000 & 0.9176659000 & -2.2635960000 \\ \mathrm{H} & 1.5564098000 & -0.6273742000 & 3.9153824000 \\ \mathrm{H} & 4.1537162000 & 0.4224472000 & -0.2767992000 \\ \mathrm{H} & 4.0479269000 & -0.4375584000 & 3.9648100000 \\ \mathrm{H} & 5.3233873000 & 0.1107894000 & 1.9196825000 \\ \mathrm{H} & -2.1724987000 & 1.6639022000 & -4.2942235000 \\ \mathrm{H} & 1.8877639000 & -1.1656557000 & -5.0172271000 \\ \mathrm{H} & -1.2643736000 & 1.3357958000 & -6.5701739000\end{array}$

31

SO_TCC (SPh)

$\begin{array}{llll}C & -0.0764638290 & -0.1447186480 & -0.0603681170 \\ C & -0.1427551460 & 0.1138451060 & 1.4153420920\end{array}$




$\begin{array}{lrrr}\text { N } & 1.2398114920 & -0.1648248500 & -0.4641528850 \\ \text { C } & -1.1098895130 & -0.3955600640 & -0.9672569170 \\ \text { C } & 1.2930527810 & 0.0995674120 & 1.8178868350 \\ \text { C } & 2.0902221980 & -0.0274292380 & 0.6440468590 \\ \text { C } & -2.4820549830 & -0.3922963960 & -0.7254138350 \\ \text { C } & 1.9118986310 & 0.2444429610 & 3.0530632140 \\ \text { C } & 3.4861646430 & -0.0150537990 & 0.6815258360 \\ \text { C } & -3.2554898720 & 0.1756585000 & 0.2933955990 \\ \text { C } & 3.3093661400 & 0.2486128060 & 3.0989518960 \\ \text { C } & 4.0782206150 & 0.1225739050 & 1.9382348950 \\ \text { C } & -2.8731443480 & 1.2851518010 & 1.1495625550 \\ \text { C } & -4.6010034260 & -0.3157965450 & 0.4047505730 \\ \text { O } & -1.7906958580 & 1.9194804690 & 1.0114547740 \\ \text { C } & -3.8170858220 & 1.7284881480 & 2.1571520350 \\ \text { C } & -5.4634214710 & 0.1481356820 & 1.3608551930 \\ \text { C } & -5.0610070970 & 1.1774908720 & 2.2524167890 \\ \text { H } & -5.7773832900 & 1.5278713940 & 3.0077520870 \\ \text { H } & -0.6242147360 & 1.1403130630 & 1.5151376280 \\ \text { H } & -0.7670885200 & -0.6308168920 & 1.9378507760 \\ \text { H } & 1.5568133880 & -0.2534711340 & -1.4173590150 \\ \text { H } & -0.7953916970 & -0.6992266810 & -1.9770530260 \\ \text { H } & -3.0667428980 & -0.9510405320 & -1.4948237990 \\ \text { H } & 1.3179189530 & 0.3494483940 & 3.9644952760 \\ \text { H } & 4.0812500880 & -0.0987157560 & -0.2294768700 \\ \text { H } & 3.8141652730 & 0.3537250960 & 4.0653027190 \\ \text { H } & 5.1717122860 & 0.1345341180 & 2.0135341900 \\ \text { H } & -4.9269524890 & -1.1126449370 & -0.2834831950 \\ \text { H } & -3.4918211380 & 2.5367060120 & 2.8152042820 \\ \text { H } & -6.4734040940 & -0.2643214700 & 1.4498607340\end{array}$

31

SO_TTC (SPh)

$\begin{array}{lrrr}C & -0.0408810600 & -0.0026851000 & 0.0350243700 \\ C & -0.0184282420 & -0.0038833600 & 1.5596372510 \\ \text { N } & 1.2555094980 & -0.0018558150 & -0.4359478660 \\ C & -1.1441556500 & -0.0024045270 & -0.7964155550 \\ C & 1.4533637690 & -0.0035936220 & 1.8577079580 \\ C & 2.1679837410 & -0.0023714200 & 0.6269364720 \\ C & -2.4496726380 & -0.0032618460 & -0.2700678510 \\ C & 2.1429564030 & -0.0042869440 & 3.0596464170 \\ C & 3.5634718750 & -0.0018447750 & 0.5816977380 \\ C & -3.5755785640 & -0.0029746460 & -1.0793752730 \\ C & 3.5424685590 & -0.0037678270 & 3.0197568630 \\ C & 4.2341164790 & -0.0025797670 & 1.8065801850\end{array}$




\begin{tabular}{|c|c|c|c|}
\hline$C$ & -3.4772804000 & -0.0017672560 & -2.5470683270 \\
\hline C & -4.8776871690 & -0.0038854170 & -0.4763657470 \\
\hline 0 & -2.3807964150 & -0.0009203720 & -3.1377829560 \\
\hline C & -4.7272165180 & -0.0015985710 & -3.3005444600 \\
\hline C & -6.0108914230 & -0.0036587570 & -1.2397567170 \\
\hline C & -5.9287151180 & -0.0025072890 & -2.6654702990 \\
\hline $\mathrm{H}$ & -6.8680495670 & -0.0023774120 & -3.2355912340 \\
\hline $\mathrm{H}$ & -0.5115846190 & 0.9054563960 & 1.9500132320 \\
\hline $\mathrm{H}$ & -0.5109291790 & -0.9141904270 & 1.9485826530 \\
\hline $\mathrm{H}$ & 1.5081062130 & -0.0009792010 & -1.4119036680 \\
\hline $\mathrm{H}$ & -1.0327989840 & -0.0015065100 & -1.8773096970 \\
\hline $\mathrm{H}$ & -2.5907129290 & -0.0041999560 & 0.8292345360 \\
\hline $\mathrm{H}$ & 1.6093685770 & -0.0052161110 & 4.0142418470 \\
\hline $\mathrm{H}$ & 4.0996935630 & -0.0008909550 & -0.3677052360 \\
\hline $\mathrm{H}$ & 4.1050794100 & -0.0042991650 & 3.9589976790 \\
\hline $\mathrm{H}$ & 5.3302552650 & -0.0022073240 & 1.8130052510 \\
\hline $\mathrm{H}$ & -4.9465688400 & -0.0047794830 & 0.6227136380 \\
\hline $\mathrm{H}$ & -4.6449500640 & -0.0007148620 & -4.3889591630 \\
\hline $\mathrm{H}$ & -7.0014984440 & -0.0043574070 & -0.7731212900 \\
\hline
\end{tabular}

31

SO_CCT (SPh)

$\begin{array}{lrrr}C & -0.0502543060 & 0.0446771850 & -0.0847468170 \\ \mathrm{C} & -0.0298312120 & -0.0875860180 & 1.4364606920 \\ \mathrm{~N} & 1.2424465860 & 0.0720880510 & -0.5561243420 \\ \mathrm{C} & -1.2012874280 & 0.2277511820 & -0.8217160170 \\ \mathrm{C} & 1.4407712220 & -0.0864889940 & 1.7359767400 \\ \mathrm{C} & 2.1524925800 & 0.0212380650 & 0.5087387130 \\ \mathrm{C} & -1.2413846240 & 0.3679327220 & -2.2320160010 \\ \mathrm{C} & 2.1322445930 & -0.1675185520 & 2.9340324670 \\ \mathrm{C} & 3.5474759510 & 0.0465343850 & 0.4618409590 \\ \mathrm{C} & -0.4028454480 & -0.2421459130 & -3.1457173370 \\ \mathrm{C} & 3.5316534510 & -0.1397936050 & 2.8931832620 \\ \mathrm{C} & 4.2206536180 & -0.0355918860 & 1.6827037820 \\ \mathrm{C} & -0.4634692650 & 0.2629366730 & -4.5318434340 \\ \mathrm{C} & 0.4235670320 & -1.3788995980 & -2.8613810510 \\ \mathrm{O} & -1.2057055250 & 1.2126503510 & -4.8269524240 \\ \mathrm{C} & 0.4233140920 & -0.3569535620 & -5.5134867640 \\ \mathrm{C} & 1.2025969680 & -1.9338836760 & -3.8383344700 \\ \mathrm{C} & 1.2245997500 & -1.3955600130 & -5.1632518710 \\ \mathrm{H} & 1.8945285090 & -1.8648017470 & -5.8970062290 \\ \mathrm{H} & -0.5441595360 & 0.7689329160 & 1.9097780410 \\ \mathrm{H} & -0.5077476720 & -1.0393006450 & 1.7360921300 \\ \mathrm{H} & 1.4910255380 & 0.2675579580 & -1.5153481280\end{array}$




$\begin{array}{lrrr}\mathrm{H} & -2.1389766900 & 0.3477123040 & -0.2765935730 \\ \mathrm{H} & -2.0197197510 & 1.0355144940 & -2.6373459320 \\ \mathrm{H} & 1.6003370820 & -0.2492479230 & 3.8858642040 \\ \mathrm{H} & 4.0802391470 & 0.1283287690 & -0.4854772510 \\ \mathrm{H} & 4.0960422950 & -0.2016456680 & 3.8292288670 \\ \mathrm{H} & 5.3164112480 & -0.0163670540 & 1.6881117880 \\ \mathrm{H} & 0.3615756990 & -1.8473257530 & -1.8714576520 \\ \mathrm{H} & 0.3906263610 & 0.0388342190 & -6.5297256730 \\ \mathrm{H} & 1.8072112330 & -2.8227709550 & -3.6295570750\end{array}$

31

SO_CTC (SPh)

$\begin{array}{lrrr}\mathrm{C} & 0.0284826590 & -0.0024781830 & -0.0787243040 \\ \mathrm{C} & -0.0410292710 & -0.0056186150 & 1.4450450100 \\ \mathrm{~N} & 1.3489766030 & -0.0000089250 & -0.4720404050 \\ \mathrm{C} & -1.0836672840 & -0.0023072260 & -0.8989593740 \\ \mathrm{C} & 1.4092916420 & -0.0045762050 & 1.8319549070 \\ \mathrm{C} & 2.1963497180 & -0.0011783380 & 0.6462148220 \\ \mathrm{C} & -0.9714543250 & 0.0004792570 & -2.3022972510 \\ \mathrm{C} & 2.0279779510 & -0.0062762100 & 3.0719431990 \\ \mathrm{C} & 3.5918320250 & 0.0005721690 & 0.6822413600 \\ \mathrm{C} & -2.0778213250 & 0.0005207830 & -3.1385693040 \\ \mathrm{C} & 3.4274475290 & -0.0045774720 & 3.1144855310 \\ \mathrm{C} & 4.1896808680 & -0.0012163840 & 1.9444650970 \\ \mathrm{C} & -3.4493927120 & -0.0022968150 & -2.6074919700 \\ \mathrm{C} & -1.8890596210 & 0.0033415880 & -4.5608727850 \\ \mathrm{O} & -3.6856541680 & -0.0048398020 & -1.3848009470 \\ \mathrm{C} & -4.5404702260 & -0.0020102960 & -3.5767285350 \\ \mathrm{C} & -2.9550150910 & 0.0034297970 & -5.4156606930 \\ \mathrm{C} & -4.2916555400 & 0.0007249530 & -4.9128165920 \\ \mathrm{H} & -5.1154630750 & 0.0009248660 & -5.6399079960 \\ \mathrm{H} & -0.5622055580 & 0.9009740080 & 1.8022537820 \\ \mathrm{H} & -0.5599398970 & -0.9149407790 & 1.7985965220 \\ \mathrm{H} & 1.6674115740 & 0.0023081150 & -1.4280024440 \\ \mathrm{H} & -2.0818025600 & -0.0044118700 & -0.4705354360 \\ \mathrm{H} & 0.0362220730 & 0.0026985600 & -2.7707432270 \\ \mathrm{H} & 1.4389338000 & -0.0088768260 & 3.9930436660 \\ \mathrm{H} & 4.1836883070 & 0.0032054880 & -0.2339106560 \\ \mathrm{H} & 3.9338267020 & -0.0058959360 & 4.0852162700 \\ \mathrm{H} & 5.2835022980 & 0.0000597970 & 2.0151817850 \\ \mathrm{H} & -0.8600832350 & 0.0054432830 & -4.9536750850 \\ \mathrm{H} & -5.5550368600 & -0.0040913700 & -3.1742419650 \\ \mathrm{H} & -2.8042616690 & 0.0055791870 & -6.5002044020\end{array}$


31

SO_TCT (SPh)

$\begin{array}{lrrr}\mathrm{C} & -0.0600968050 & -0.1199048110 & -0.0338479330 \\ \mathrm{C} & -0.1151375380 & -0.1687345370 & 1.4904370290 \\ \mathrm{~N} & 1.2652146240 & -0.0243790360 & -0.4337544700 \\ \mathrm{C} & -1.0801296950 & -0.3344509650 & -0.9310259820 \\ \mathrm{C} & 1.3369375710 & -0.0949684970 & 1.8646117090 \\ \mathrm{C} & 2.1159554290 & -0.0037953720 & 0.6780268860 \\ \mathrm{C} & -2.4525107620 & -0.4384607420 & -0.5722874870 \\ \mathrm{C} & 1.9583890610 & -0.0937038260 & 3.1028834990 \\ \mathrm{C} & 3.5083291030 & 0.0892274300 & 0.7117928330 \\ \mathrm{C} & -3.0940537840 & 0.3366428220 & 0.3709338500 \\ \mathrm{C} & 3.3554127150 & -0.0025494210 & 3.1431880700 \\ \mathrm{C} & 4.1105449290 & 0.0873623770 & 1.9719589960 \\ \mathrm{C} & -4.4411167180 & -0.0897378240 & 0.7965999690 \\ \mathrm{C} & -2.5615078030 & 1.5627956480 & 0.8854687300 \\ \mathrm{O} & -4.9561705480 & -1.1198786700 & 0.3363797260 \\ \mathrm{C} & -5.1057652350 & 0.7138562600 & 1.8227033040 \\ \mathrm{C} & -3.2530132990 & 2.2854620000 & 1.8181863700 \\ \mathrm{C} & -4.5180510260 & 1.8343085850 & 2.3128240670 \\ \mathrm{H} & -5.0107573770 & 2.4390045980 & 3.0864633680 \\ \mathrm{H} & -0.6787106410 & 0.6764485660 & 1.9157637770 \\ \mathrm{H} & -0.5729135640 & -1.1257391510 & 1.8000500360 \\ \mathrm{H} & 1.5724746740 & 0.0610839310 & -1.3884445930 \\ \mathrm{H} & -0.8256480170 & -0.5025176200 & -1.9797615470 \\ \mathrm{H} & -3.0482149200 & -1.1989670090 & -1.1036173440 \\ \mathrm{H} & 1.3741086730 & -0.1649956890 & 4.0240900530 \\ \mathrm{H} & 4.0941570060 & 0.1575564500 & -0.2050314900 \\ \mathrm{H} & 3.8643389800 & -0.0015630910 & 4.1122220310 \\ \mathrm{H} & 5.2024203500 & 0.1562006840 & 2.0398578140 \\ \mathrm{H} & -1.6286874600 & 1.9533455640 & 0.4545546320 \\ \mathrm{H} & -6.0903959910 & 0.3784213480 & 2.1530544970 \\ \mathrm{H} & -2.8635748470 & 3.2409781310 & 2.1859711550\end{array}$

31

SO_CCC_H8 (SPh)

$\begin{array}{lrrr}\mathrm{C} & 0.5067115620 & 1.4771537330 & -0.2739221420 \\ \mathrm{C} & 1.8320024490 & 1.8693134710 & -0.5121932530 \\ \mathrm{~N} & 0.4921792690 & 0.1947575430 & 0.2777589480 \\ \mathrm{C} & -0.6619916020 & 2.2900080580 & -0.5229518350 \\ \mathrm{C} & 2.6682698540 & 0.7835559530 & -0.0986381100 \\ \mathrm{C} & 1.7911239930 & -0.2479809480 & 0.3961773030 \\ \mathrm{C} & -1.9445298760 & 1.8873027700 & -0.4175201480 \\ \mathrm{C} & 4.0552262360 & 0.5750692800 & -0.0866879530\end{array}$




$\begin{array}{lrrr}\text { C } & 2.2902355250 & -1.4653609980 & 0.8909238310 \\ \text { C } & -2.3643813360 & 0.5083144590 & -0.1133572280 \\ \text { C } & 4.5338499990 & -0.6310768100 & 0.4062260210 \\ \text { C } & 3.6696833700 & -1.6302753400 & 0.8854620660 \\ \text { C } & -2.1902998410 & -0.5023804360 & -1.0817305280 \\ \text { O } & -2.9963868770 & 0.1946643750 & 1.0926922550 \\ \text { C } & -1.6049763920 & -0.2690587670 & -2.2822608930 \\ \text { C } & -2.6407649970 & -1.8156069610 & -0.8618173020 \\ \text { C } & -3.4371196700 & -1.1094500730 & 1.3224512350 \\ \text { H } & -3.2625801540 & -2.0984427260 & 0.3507101480 \\ \text { H } & -3.6179409610 & -3.1169970410 & 0.5463266740 \\ \text { H } & -1.2038528630 & 0.6454596100 & -2.2719555320 \\ \text { H } & 2.1578996950 & 2.8089580230 & -0.9322406430 \\ \text { H } & -0.3353185070 & -0.3066697540 & 0.5629928200 \\ \text { H } & -0.4388417580 & 3.3290130480 & -0.8064884380 \\ \text { H } & -2.7670177530 & 2.5940824040 & -0.5753584850 \\ \text { H } & 4.7334158700 & 1.3489534350 & -0.4587413100 \\ \text { H } & 1.6145981320 & -2.2404658630 & 1.2589056800 \\ \text { H } & 5.6145914910 & -0.8158514850 & 0.4254312530 \\ \text { H } & 4.0988191310 & -2.5655115140 & 1.2655295560 \\ \text { H } & -3.1337838400 & 0.9673273450 & 1.8547833720 \\ \text { H } & -2.5098693670 & -2.5658938390 & -1.6373923080 \\ & -3.9224247820 & -1.3569706660 & 2.2705593420\end{array}$

31

SO_CCC_H9 (SPh)

$\begin{array}{lrrr}C & -0.1413283470 & -0.0360750600 & -0.0752211590 \\ C & -0.0747158920 & 0.7101230110 & 1.2637943060 \\ \mathrm{~N} & 1.0193019080 & -0.3394358780 & -0.5752328210 \\ \mathrm{C} & -1.4179602710 & -0.3922748830 & -0.6706243050 \\ \mathrm{C} & 1.4075496640 & 0.8166697280 & 1.4312738080 \\ \mathrm{C} & 2.0004805220 & 0.1722489880 & 0.3074174730 \\ \mathrm{C} & -1.6468094900 & -0.4604625190 & -2.0046027930 \\ \mathrm{C} & 2.1982576070 & 1.3836854930 & 2.4185595030 \\ \mathrm{C} & 3.3852348620 & 0.0950929140 & 0.1620168190 \\ \mathrm{C} & -0.7061454000 & -0.0832342210 & -3.0655562950 \\ \mathrm{C} & 3.5873750790 & 1.3029743240 & 2.2688560160 \\ \mathrm{C} & 4.1671111560 & 0.6722108720 & 1.1660964340 \\ \mathrm{C} & 0.4571660510 & -0.8143059080 & -3.3817354730 \\ \mathrm{C} & -1.0390463670 & 1.0320847530 & -3.8501092820 \\ \mathrm{O} & 0.7916654630 & -1.9435207860 & -2.7145506590 \\ \mathrm{C} & 1.2684528940 & -0.4452678850 & -4.4694338560 \\ \mathrm{C} & -0.2267809370 & 1.4039731720 & -4.9193702320 \\ \mathrm{C} & 0.9130242050 & 0.6610671050 & -5.2364686530\end{array}$




$\begin{array}{lrrr}\text { H } & 1.5363705120 & 0.9579407350 & -6.0880967690 \\ \mathrm{H} & -0.5527808390 & 1.6971008140 & 1.1716513400 \\ \mathrm{H} & -0.5376673750 & 0.1154062690 & 2.0660029790 \\ \mathrm{H} & 1.0003505930 & -1.6609481190 & -1.7711246780 \\ \mathrm{H} & -2.2230780920 & -0.6103525380 & 0.0402805160 \\ \mathrm{H} & -2.6416729620 & -0.7655112440 & -2.3655625610 \\ \mathrm{H} & 1.7532744000 & 1.8771042170 & 3.2876208110 \\ \mathrm{H} & 3.8273597140 & -0.3942212500 & -0.7068002070 \\ \mathrm{H} & 4.2343175840 & 1.7475248060 & 3.0342279760 \\ \mathrm{H} & 5.2592243410 & 0.6314879490 & 1.0839744480 \\ \mathrm{H} & -1.9421589650 & 1.6110856880 & -3.6224249160 \\ \mathrm{H} & 2.1552571790 & -1.0364510420 & -4.6978656580 \\ \mathrm{H} & -0.4881134320 & 2.2834166790 & -5.5171859940\end{array}$

31

SO_TCC_H8 (SPh)

$\begin{array}{lrrr}\mathrm{C} & -0.1275281980 & -0.2076335430 & -0.1222715500 \\ \mathrm{C} & -0.2859397500 & -0.1845660330 & 1.2669056000 \\ \mathrm{~N} & 1.2413228970 & -0.1884246980 & -0.4363841980 \\ \mathrm{C} & -1.1092768620 & -0.3142923140 & -1.1775782350 \\ \mathrm{C} & 1.0309710980 & -0.1389052740 & 1.8347008250 \\ \mathrm{C} & 1.9643240670 & -0.1394865060 & 0.7376478710 \\ \mathrm{C} & -2.4416228600 & -0.1675696770 & -1.0187075460 \\ \mathrm{C} & 1.5142841790 & -0.1013319260 & 3.1493291520 \\ \mathrm{C} & 3.3520164830 & -0.0905205230 & 0.9470835030 \\ \mathrm{C} & -3.0977253260 & 0.2487938290 & 0.2300856870 \\ \mathrm{C} & 2.8886853780 & -0.0566035290 & 3.3442374520 \\ \mathrm{C} & 3.7889388850 & -0.0536802030 & 2.2657570950 \\ \mathrm{C} & -2.8244955350 & 1.5107462200 & 0.7969583800 \\ \mathrm{C} & -4.0472447490 & -0.5777562890 & 0.8379028190 \\ \mathrm{O} & -1.9484676270 & 2.3905357750 & 0.2505343980 \\ \mathrm{C} & -3.4998641980 & 1.9612226830 & 1.9446249870 \\ \mathrm{C} & -4.7112132580 & -0.1430258350 & 1.9855281100 \\ \mathrm{C} & -4.4393416530 & 1.1158316610 & 2.5270854720 \\ \mathrm{H} & -4.9681754580 & 1.4420995940 & 3.4307238840 \\ \mathrm{H} & -1.3608749460 & 1.9042595020 & -0.3921447460 \\ \mathrm{H} & -1.2162516490 & -0.2141164040 & 1.8076586450 \\ \mathrm{H} & 1.6270288820 & -0.1928272240 & -1.3664537370 \\ \mathrm{H} & -0.7072037220 & -0.5606704260 & -2.1730150240 \\ \mathrm{H} & -3.1209100790 & -0.3423765000 & -1.8624236710 \\ \mathrm{H} & 0.8181229910 & -0.1039565160 & 3.9932145240 \\ \mathrm{H} & 4.0490337430 & -0.0821069430 & 0.1057386620 \\ \mathrm{H} & 3.2887647600 & -0.0231419100 & 4.3644387570 \\ \mathrm{H} & 4.8657763570 & -0.0213986470 & 2.4723299890\end{array}$




$\begin{array}{lrrr}H & -4.2606073320 & -1.5662844410 & 0.4187520270 \\ H & -3.2884594590 & 2.9528418410 & 2.3375459310 \\ H & -5.4449667030 & -0.7967202860 & 2.4661719290\end{array}$

31

SO_TCC_H9 (SPh)

$\begin{array}{lrrr}\mathrm{C} & -0.4693105000 & 0.7065356000 & 1.2047245000 \\ \mathrm{C} & -1.2871595000 & 1.5965496000 & 0.2521951000 \\ \mathrm{~N} & -1.0203350000 & -0.4270910000 & 1.4967578000 \\ \mathrm{C} & 0.7695147000 & 1.2230611000 & 1.7939157000 \\ \mathrm{C} & -2.5021365000 & 0.7386942000 & 0.0924132000 \\ \mathrm{C} & -2.2713892000 & -0.4550406000 & 0.8382131000 \\ \mathrm{C} & 2.0372347000 & 1.0557559000 & 1.3826046000 \\ \mathrm{C} & -3.6774783000 & 0.9182798000 & -0.6195013000 \\ \mathrm{C} & -3.2278416000 & -1.4707532000 & 0.8866375000 \\ \mathrm{C} & 2.5778080000 & 0.3271917000 & 0.2349918000 \\ \mathrm{C} & -4.6273061000 & -0.1088689000 & -0.5723286000 \\ \mathrm{C} & -4.4084657000 & -1.2734466000 & 0.1665578000 \\ \mathrm{C} & 1.8527028000 & -0.3260753000 & -0.7847458000 \\ \mathrm{C} & 3.9837141000 & 0.2799048000 & 0.1532778000 \\ \mathrm{O} & 0.4974341000 & -0.2245872000 & -0.8631722000 \\ \mathrm{C} & 2.4996437000 & -1.0033439000 & -1.8332063000 \\ \mathrm{C} & 4.6300309000 & -0.3932390000 & -0.8781516000 \\ \mathrm{C} & 3.8894283000 & -1.0376444000 & -1.8719884000 \\ \mathrm{H} & 4.4039500000 & -1.5652544000 & -2.6827231000 \\ \mathrm{H} & -1.5075271000 & 2.5714640000 & 0.7156357000 \\ \mathrm{H} & -0.7398443000 & 1.7110074000 & -0.6931713000 \\ \mathrm{H} & 0.1086566000 & -1.0377067000 & -0.4407879000 \\ \mathrm{H} & 0.5709394000 & 1.8029443000 & 2.7073183000 \\ \mathrm{H} & 2.8263380000 & 1.5175017000 & 2.0105665000 \\ \mathrm{H} & -3.8617257000 & 1.8282583000 & -1.1976168000 \\ \mathrm{H} & -3.0521861000 & -2.3755517000 & 1.4708752000 \\ \mathrm{H} & -5.5635583000 & 0.0058826000 & -1.1318865000 \\ \mathrm{H} & -5.1823873000 & -2.0505655000 & 0.1825948000 \\ \mathrm{H} & 4.5843286000 & 0.7860463000 & 0.9228244000 \\ \mathrm{H} & 1.9014067000 & -1.4923264000 & -2.6034818000 \\ \mathrm{H} & 5.7237227000 & -0.4179643000 & -0.9112144000\end{array}$

31

SO_CCT_H8 (SPh)

$\begin{array}{llll}\mathrm{C} & 0.6682113350 & 1.3767617210 & -0.4732119310 \\ \mathrm{C} & 1.8645502110 & 1.9797630230 & -0.0692764310 \\ \mathrm{~N} & 0.7149707740 & 0.0104432680 & -0.1889344650 \\ \mathrm{C} & -0.4310410590 & 2.0358929600 & -1.1477608730\end{array}$




\begin{tabular}{|c|c|c|c|}
\hline C & 2.6796401350 & 0.9492160430 & 0.5019196900 \\
\hline C & 1.9301372150 & -0.2781132850 & 0.3965681860 \\
\hline C & -1.7024866750 & 1.5913694400 & -1.2237183800 \\
\hline C & 3.9575967970 & 0.9262129600 & 1.0755060390 \\
\hline C & 2.4447701300 & -1.5001182320 & 0.8583270360 \\
\hline C & -2.2231745310 & 0.4107526240 & -0.5260339780 \\
\hline C & 4.4554878650 & -0.2890690080 & 1.5268962690 \\
\hline C & 3.7157488970 & -1.4782761510 & 1.4180857560 \\
\hline C & -3.0976474120 & -0.4706025450 & -1.1998260550 \\
\hline C & -1.9710152950 & 0.1801084830 & 0.8314697680 \\
\hline 0 & -3.3444982530 & -0.1714476770 & -2.5044319890 \\
\hline C & -3.6573287470 & -1.5901717090 & -0.5690011560 \\
\hline C & -2.5308487700 & -0.9268893580 & 1.4714171480 \\
\hline C & -3.3650041160 & -1.8042395740 & 0.7771214740 \\
\hline $\mathrm{H}$ & -3.8014809140 & -2.6673582410 & 1.2926305210 \\
\hline $\mathrm{H}$ & -3.9322742030 & -0.8714886870 & -2.8986830050 \\
\hline $\mathrm{H}$ & 2.1187947860 & 3.0240686080 & -0.1587949060 \\
\hline $\mathrm{H}$ & -0.0012905660 & -0.6557962050 & -0.4310564220 \\
\hline $\mathrm{H}$ & -0.1538180590 & 2.9752866370 & -1.6481128960 \\
\hline $\mathrm{H}$ & -2.4416196600 & 2.1238222360 & -1.8332375830 \\
\hline $\mathrm{H}$ & 4.5419473090 & 1.8477754660 & 1.1599330890 \\
\hline $\mathrm{H}$ & 1.8644743290 & -2.4221689670 & 0.7739001570 \\
\hline $\mathrm{H}$ & 5.4545923730 & -0.3294771170 & 1.9777435550 \\
\hline $\mathrm{H}$ & 4.1571442170 & -2.4138353540 & 1.7832886800 \\
\hline $\mathrm{H}$ & -1.3306692960 & 0.8709744710 & 1.3852630810 \\
\hline $\mathrm{H}$ & -4.3061409330 & -2.2735956640 & -1.1199722150 \\
\hline $\mathrm{H}$ & -2.3236699190 & -1.0990049650 & 2.5309347260 \\
\hline
\end{tabular}

31

SO_CCT_H9 (SPh)

$\begin{array}{lrrr}C & -0.7893181000 & 1.1508566000 & 0.2346509000 \\ C & -2.1180552000 & 1.8768532000 & 0.5233723000 \\ \mathrm{~N} & -0.8976410000 & -0.1434063000 & 0.2221045000 \\ \mathrm{C} & 0.3861732000 & 1.9753102000 & 0.0133868000 \\ \mathrm{C} & -3.0263976000 & 0.7060306000 & 0.7204046000 \\ \mathrm{C} & -2.2422147000 & -0.4648275000 & 0.5060838000 \\ \mathrm{C} & 1.6437238000 & 1.6350765000 & -0.3534348000 \\ \mathrm{C} & -4.3737717000 & 0.6139046000 & 1.0317412000 \\ \mathrm{C} & -2.8107592000 & -1.7347768000 & 0.5945120000 \\ \mathrm{C} & 2.2662315000 & 0.3425155000 & -0.5875099000 \\ \mathrm{C} & -4.9323105000 & -0.6637068000 & 1.1380789000 \\ \mathrm{C} & -4.1686097000 & -1.8106537000 & 0.9184890000 \\ \mathrm{C} & 3.5062773000 & 0.3668666000 & -1.2773238000 \\ \mathrm{C} & 1.8037983000 & -0.8994813000 & -0.1335478000\end{array}$




$\begin{array}{lrrr}\text { O } & 4.0302972000 & 1.5753814000 & -1.6562923000 \\ \mathrm{C} & 4.2766050000 & -0.7800378000 & -1.4968299000 \\ \mathrm{C} & 2.5694617000 & -2.0459668000 & -0.3510507000 \\ \mathrm{C} & 3.7947271000 & -1.9942484000 & -1.0122818000 \\ \mathrm{H} & 4.3766293000 & -2.9111094000 & -1.1596923000 \\ \mathrm{H} & -2.0329198000 & 2.5046686000 & 1.4245332000 \\ \mathrm{H} & -2.4207029000 & 2.4761436000 & -0.3506622000 \\ \mathrm{H} & 3.6039934000 & 1.8175520000 & -2.5216587000 \\ \mathrm{H} & 0.1833301000 & 3.0511004000 & 0.1567958000 \\ \mathrm{H} & 2.3458299000 & 2.4780200000 & -0.5042126000 \\ \mathrm{H} & -4.9819849000 & 1.5086544000 & 1.1943492000 \\ \mathrm{H} & -2.2128817000 & -2.6283881000 & 0.4073935000 \\ \mathrm{H} & -5.9955824000 & -0.7609273000 & 1.3914086000 \\ \mathrm{H} & -4.6404129000 & -2.7957462000 & 1.0083100000 \\ \mathrm{H} & 0.8519516000 & -0.9717220000 & 0.3804025000 \\ \mathrm{H} & 5.2380236000 & -0.7100602000 & -2.0063144000 \\ \mathrm{H} & 2.1948612000 & -3.0082856000 & 0.0149240000\end{array}$

31

SO_TCT_H8 (SPh)

$\begin{array}{lrrr}\mathrm{C} & 0.6034897000 & 0.7725491000 & 0.9938964000 \\ \mathrm{C} & 0.8125486000 & -0.2862553000 & 0.1069008000 \\ \mathrm{~N} & 1.7744597000 & 1.5408637000 & 1.0922906000 \\ \mathrm{C} & -0.5279879000 & 1.0745849000 & 1.8434637000 \\ \mathrm{C} & 2.1587854000 & -0.1611126000 & -0.3789868000 \\ \mathrm{C} & 2.7253532000 & 1.0054911000 & 0.2478021000 \\ \mathrm{C} & -1.8017075000 & 0.6903404000 & 1.6163278000 \\ \mathrm{C} & 2.9420449000 & -0.9069981000 & -1.2701900000 \\ \mathrm{C} & 4.0368667000 & 1.4284274000 & -0.0201468000 \\ \mathrm{C} & -2.2839349000 & 0.0444326000 & 0.3884828000 \\ \mathrm{C} & 4.2385335000 & -0.4798123000 & -1.5279310000 \\ \mathrm{C} & 4.7744081000 & 0.6655039000 & -0.9169407000 \\ \mathrm{C} & -3.2001179000 & -1.0248139000 & 0.4536858000 \\ \mathrm{C} & -1.9195368000 & 0.5419092000 & -0.8680990000 \\ \mathrm{O} & -3.6123991000 & -1.5861122000 & 1.6230227000 \\ \mathrm{C} & -3.7384671000 & -1.6153828000 & -0.7020360000 \\ \mathrm{C} & -2.4423884000 & -0.0418956000 & -2.0215775000 \\ \mathrm{C} & -3.3525235000 & -1.0985010000 & -1.9358218000 \\ \mathrm{H} & -3.7710896000 & -1.5272909000 & -2.8539783000 \\ \mathrm{H} & -3.1319142000 & -1.1575031000 & 2.3809231000 \\ \mathrm{H} & 0.1018697000 & -1.0532409000 & -0.1506257000 \\ \mathrm{H} & 1.8752815000 & 2.3839021000 & 1.6334464000 \\ \mathrm{H} & -0.2814854000 & 1.6333318000 & 2.7621184000 \\ \mathrm{H} & -2.5762976000 & 0.9230662000 & 2.3630951000\end{array}$




$\begin{array}{lrrr}\mathrm{H} & 2.5367897000 & -1.8058269000 & -1.7431199000 \\ \mathrm{H} & 4.4495806000 & 2.3199251000 & 0.4580018000 \\ \mathrm{H} & 4.8652365000 & -1.0463438000 & -2.2273064000 \\ \mathrm{H} & 5.8031298000 & 0.9631962000 & -1.1526613000 \\ \mathrm{H} & -1.2238040000 & 1.3813674000 & -0.9398385000 \\ \mathrm{H} & -4.4328944000 & -2.4468749000 & -0.6113455000 \\ \mathrm{H} & -2.1489907000 & 0.3480384000 & -2.9999791000\end{array}$

31

SO_TCT_H9 (SPh)

$\begin{array}{lrrr}\text { C } & -0.5676790000 & 0.8107272000 & 0.9616565000 \\ \text { C } & -1.3840304000 & 0.7269089000 & -0.3407446000 \\ \text { N } & -1.0682779000 & 0.1524338000 & 1.9571386000 \\ \text { C } & 0.5629804000 & 1.7405103000 & 1.0442968000 \\ \text { C } & -2.5538003000 & -0.0712872000 & 0.1447073000 \\ \text { C } & -2.2941456000 & -0.3973719000 & 1.5096367000 \\ \text { C } & 1.7230609000 & 1.6549723000 & 0.3743768000 \\ \text { C } & -3.7448558000 & -0.4475989000 & -0.4575786000 \\ \text { C } & -3.2176319000 & -1.1157258000 & 2.2686849000 \\ \text { C } & 2.1601350000 & 0.5415056000 & -0.4600937000 \\ \text { C } & -4.6692816000 & -1.1611282000 & 0.3141830000 \\ \text { C } & -4.4116112000 & -1.4894905000 & 1.6471589000 \\ \text { C } & 3.1345289000 & 0.7788890000 & -1.4605159000 \\ \text { C } & 1.7899958000 & -0.7895900000 & -0.2196132000 \\ \text { O } & 3.4828770000 & 2.0821929000 & -1.6503298000 \\ \text { C } & 3.7394645000 & -0.2604019000 & -2.1774949000 \\ \text { C } & 2.3894874000 & -1.8308112000 & -0.9277640000 \\ \text { C } & 3.3688978000 & -1.5726296000 & -1.8859155000 \\ \text { H } & 3.8558697000 & -2.4016461000 & -2.4096016000 \\ \text { H } & -1.6743421000 & 1.7396707000 & -0.6622935000 \\ \text { H } & -0.8060719000 & 0.2153890000 & -1.1248251000 \\ \text { H } & 4.0532407000 & 2.1492273000 & -2.4630913000 \\ \text { H } & 0.3619201000 & 2.5907087000 & 1.7076079000 \\ \text { H } & 2.4627272000 & 2.4598388000 & 0.4814555000 \\ \text { H } & -3.9711101000 & -0.1872619000 & -1.4961808000 \\ \text { H } & -3.0119074000 & -1.3572999000 & 3.3125270000 \\ \text { H } & -5.6289299000 & -1.4514752000 & -0.1307467000 \\ \text { H } & -5.1687222000 & -2.0357763000 & 2.2227733000 \\ \text { H } & 1.0406480000 & -1.0129272000 & 0.5430614000 \\ \text { H } & 4.4923393000 & -0.0440309000 & -2.9386813000 \\ \text { H } & 2.1037479000 & -2.8644654000 & -0.7136050000\end{array}$

40

SO_SPmc 


\begin{tabular}{|c|c|c|c|}
\hline C & 0.1487600000 & 1.0053000000 & -0.1146100000 \\
\hline C & 1.2583400000 & 1.3852700000 & -1.1702700000 \\
\hline $\mathrm{N}$ & 0.8679700000 & 0.4617800000 & 1.0234800000 \\
\hline C & -0.8054100000 & 2.0912600000 & 0.2859300000 \\
\hline C & 2.3670300000 & 0.4391400000 & -0.7565000000 \\
\hline C & 2.0970400000 & -0.0388600000 & 0.5567600000 \\
\hline C & -2.1316100000 & 1.8959100000 & 0.3501900000 \\
\hline C & 3.5257300000 & 0.0577100000 & -1.4106700000 \\
\hline C & 2.9961300000 & -0.8752800000 & 1.2242200000 \\
\hline C & -2.6800900000 & 0.5923600000 & 0.0368700000 \\
\hline C & 4.4139000000 & -0.8060300000 & -0.7552600000 \\
\hline C & 4.1581300000 & -1.2476300000 & 0.5442700000 \\
\hline C & -1.8033800000 & -0.3904000000 & -0.4811500000 \\
\hline C & -4.0343600000 & 0.2759700000 & 0.2047100000 \\
\hline 0 & -0.5070600000 & -0.1244100000 & -0.7223200000 \\
\hline C & -2.2586900000 & -1.6832600000 & -0.7999000000 \\
\hline C & -4.4925700000 & -0.9953000000 & -0.1145800000 \\
\hline C & -3.6032100000 & -1.9677100000 & -0.6059800000 \\
\hline $\mathrm{H}$ & -3.9822900000 & -2.9694100000 & -0.8408400000 \\
\hline $\mathrm{H}$ & -0.3623600000 & 3.0605800000 & 0.5198300000 \\
\hline $\mathrm{H}$ & -2.8268200000 & 2.6962300000 & 0.6308100000 \\
\hline $\mathrm{H}$ & 3.7403300000 & 0.4178500000 & -2.4228800000 \\
\hline $\mathrm{H}$ & 2.7962200000 & -1.2143100000 & 2.2419200000 \\
\hline $\mathrm{H}$ & 5.3250900000 & -1.1323600000 & -1.2676200000 \\
\hline $\mathrm{H}$ & 4.8805000000 & -1.9062100000 & 1.0437800000 \\
\hline $\mathrm{H}$ & -4.7204900000 & 1.0441700000 & 0.5825400000 \\
\hline $\mathrm{H}$ & -1.5576500000 & -2.4206800000 & -1.1887300000 \\
\hline $\mathrm{H}$ & -5.5516200000 & -1.2419800000 & 0.0095200000 \\
\hline C & 0.2006300000 & -0.1328300000 & 2.1442500000 \\
\hline $\mathrm{H}$ & -0.7357200000 & 0.4180200000 & 2.3730200000 \\
\hline $\mathrm{H}$ & -0.0585300000 & -1.2055600000 & 1.9608900000 \\
\hline $\mathrm{H}$ & 0.8533200000 & -0.0887100000 & 3.0483000000 \\
\hline C & 0.7948700000 & 1.1925800000 & -2.6006100000 \\
\hline $\mathrm{H}$ & -0.0879500000 & 1.8178400000 & -2.8005100000 \\
\hline $\mathrm{H}$ & 1.6031500000 & 1.4830800000 & -3.2920000000 \\
\hline $\mathrm{H}$ & 0.5291900000 & 0.1467700000 & -2.7796700000 \\
\hline C & 1.7509100000 & 2.8091800000 & -0.9341100000 \\
\hline $\mathrm{H}$ & 2.6536400000 & 2.9859400000 & -1.5381600000 \\
\hline $\mathrm{H}$ & 0.9826600000 & 3.5417400000 & -1.2263000000 \\
\hline $\mathrm{H}$ & 2.0098600000 & 2.9596500000 & 0.1236800000 \\
\hline
\end{tabular}

40

SO_SPmt

$\begin{array}{llll}\text { C } & 0.0759000000 & 0.6685100000 & -0.5383800000\end{array}$ 


\begin{tabular}{|c|c|c|c|}
\hline C & 0.8149300000 & -0.1670500000 & -1.6491400000 \\
\hline $\mathrm{N}$ & 1.1319300000 & 1.2063300000 & 0.3051400000 \\
\hline C & -0.8550900000 & 1.7279400000 & -1.0549600000 \\
\hline C & 2.0964800000 & -0.5208000000 & -0.9233400000 \\
\hline C & 2.2388800000 & 0.3464500000 & 0.1971000000 \\
\hline C & -2.1877900000 & 1.6259500000 & -0.9449600000 \\
\hline C & 3.0811300000 & -1.4526800000 & -1.2104800000 \\
\hline C & 3.3682400000 & 0.2892500000 & 1.0182900000 \\
\hline C & -2.7756600000 & 0.4743100000 & -0.2929300000 \\
\hline C & 4.2039100000 & -1.5259300000 & -0.3760000000 \\
\hline C & 4.3450000000 & -0.6606800000 & 0.7095900000 \\
\hline C & -1.9055400000 & -0.4316300000 & 0.3617200000 \\
\hline C & -4.1588000000 & 0.2669800000 & -0.2191900000 \\
\hline 0 & -0.5673100000 & -0.2816200000 & 0.3358800000 \\
\hline C & -2.4094400000 & -1.5272400000 & 1.0879800000 \\
\hline C & -4.6575100000 & -0.8247100000 & 0.4812500000 \\
\hline C & -3.7839100000 & -1.7128200000 & 1.1316100000 \\
\hline $\mathrm{H}$ & -4.1985200000 & -2.5711600000 & 1.6743000000 \\
\hline $\mathrm{H}$ & -0.3785800000 & 2.5994700000 & -1.5079200000 \\
\hline $\mathrm{H}$ & -2.8645800000 & 2.4080800000 & -1.3107600000 \\
\hline $\mathrm{H}$ & 2.9819100000 & -2.1259700000 & -2.0678500000 \\
\hline $\mathrm{H}$ & 3.4818500000 & 0.9689900000 & 1.8647200000 \\
\hline $\mathrm{H}$ & 4.9804400000 & -2.2730100000 & -0.5767400000 \\
\hline $\mathrm{H}$ & 5.2376400000 & -0.7334900000 & 1.3422400000 \\
\hline $\mathrm{H}$ & -4.8376000000 & 0.9720900000 & -0.7176200000 \\
\hline $\mathrm{H}$ & -1.7191000000 & -2.2043100000 & 1.5877100000 \\
\hline $\mathrm{H}$ & -5.7363400000 & -0.9967600000 & 0.5325200000 \\
\hline C & 0.8458200000 & 1.8970900000 & 1.5297800000 \\
\hline $\mathrm{H}$ & 0.6541300000 & 1.2009000000 & 2.3811100000 \\
\hline $\mathrm{H}$ & 1.7102000000 & 2.5498600000 & 1.7988600000 \\
\hline $\mathrm{H}$ & -0.0504700000 & 2.5398200000 & 1.4012300000 \\
\hline C & 0.0135800000 & -1.3750100000 & -2.0946700000 \\
\hline $\mathrm{H}$ & -0.1525100000 & -2.0556300000 & -1.2517700000 \\
\hline $\mathrm{H}$ & -0.9571800000 & -1.0493300000 & -2.4975000000 \\
\hline $\mathrm{H}$ & 0.5629000000 & -1.9098400000 & -2.8869200000 \\
\hline C & 1.1698000000 & 0.7289900000 & -2.8312300000 \\
\hline $\mathrm{H}$ & 1.8170500000 & 0.1776400000 & -3.5284400000 \\
\hline $\mathrm{H}$ & 0.2599700000 & 1.0363700000 & -3.3659400000 \\
\hline $\mathrm{H}$ & 1.7098800000 & 1.6235800000 & -2.4889500000 \\
\hline
\end{tabular}

40

S1S0_SPmc

$\begin{array}{llll}C & 0.3001200000 & 1.0348300000 & -0.1637200000 \\ \text { C } & 1.4009900000 & 1.4338500000 & -1.1634200000\end{array}$




\begin{tabular}{lrrr} 
N & 0.8760700000 & 0.3239300000 & 0.9026000000 \\
$\mathrm{C}$ & -0.7398600000 & 2.0109100000 & 0.2139600000 \\
$\mathrm{C}$ & 2.5572400000 & 0.5774700000 & -0.6663500000 \\
$\mathrm{C}$ & 2.1844900000 & -0.0548400000 & 0.5510900000 \\
$\mathrm{C}$ & -2.0745500000 & 1.7972400000 & 0.2087500000 \\
$\mathrm{C}$ & 3.8184500000 & 0.3580500000 & -1.1967500000 \\
$\mathrm{C}$ & 3.0532800000 & -0.9070900000 & 1.2384300000 \\
$\mathrm{C}$ & -2.7282700000 & 0.5466300000 & -0.0977200000 \\
$\mathrm{C}$ & 4.6965900000 & -0.4913200000 & -0.5107400000 \\
$\mathrm{C}$ & 4.3188600000 & -1.1091200000 & 0.6822700000 \\
$\mathrm{C}$ & -2.1064800000 & -0.4972400000 & -0.9176600000 \\
$\mathrm{C}$ & -3.9949200000 & 0.3073000000 & 0.4547400000 \\
$\mathrm{O}$ & -1.0826800000 & -0.2895300000 & -1.5855800000 \\
$\mathrm{C}$ & -2.7779800000 & -1.7915000000 & -0.9860300000 \\
$\mathrm{C}$ & -4.6220600000 & -0.9228200000 & 0.3022800000 \\
$\mathrm{C}$ & -4.0031400000 & -1.9777600000 & -0.4020500000 \\
$\mathrm{H}$ & -4.5218500000 & -2.9395200000 & -0.4782500000 \\
$\mathrm{H}$ & -0.3689800000 & 2.9836000000 & 0.5871200000 \\
$\mathrm{H}$ & -2.7464200000 & 2.6103300000 & 0.5316100000 \\
$\mathrm{H}$ & 4.1227700000 & 0.8353300000 & -2.1336300000 \\
$\mathrm{H}$ & 2.7559600000 & -1.3901600000 & 2.1710900000 \\
$\mathrm{H}$ & 5.6968100000 & -0.6748900000 & -0.9195600000 \\
$\mathrm{H}$ & 5.0284000000 & -1.7699000000 & 1.1960900000 \\
$\mathrm{H}$ & -4.4954900000 & 1.0983800000 & 1.0270300000 \\
$\mathrm{H}$ & -2.2745700000 & -2.5859500000 & -1.5415100000 \\
$\mathrm{H}$ & -5.6086100000 & -1.0892600000 & 0.7501300000 \\
$\mathrm{C}$ & 0.1277900000 & -0.3391800000 & 1.9291500000 \\
$\mathrm{H}$ & -0.8657900000 & 0.1384900000 & 2.0522100000 \\
$\mathrm{H}$ & -0.0313100000 & -1.4168000000 & 1.6815400000 \\
$\mathrm{H}$ & 0.6668600000 & -0.2823100000 & 2.9052600000 \\
$\mathrm{H}$ & 1.0171500000 & 1.0809000000 & -2.5890900000 \\
$\mathrm{H}$ & 0.1252100000 & 1.6510300000 & -2.8877800000 \\
$\mathrm{H}$ & 1.8462200000 & 1.3260500000 & -3.2776700000 \\
$\mathrm{H}$ & 0.7872500000 & 0.0136300000 & -2.6577100000 \\
$\mathrm{H}$ & 1.7793100000 & 2.9085100000 & -1.0485500000 \\
$\mathrm{H}$ & 2.6510900000 & 3.1184100000 & -1.6876000000 \\
$\mathrm{H}$ & 3.9453900000 & 3.5455300000 & -1.3810400000 \\
$\mathrm{H}$ & 3.1662100000 & -0.0106300000 \\
\hline
\end{tabular}

40

S1S0_SPmt

$\begin{array}{llrr}C & 0.2403800000 & 0.8062700000 & -0.5315900000 \\ \mathrm{C} & 0.8301700000 & -0.1187600000 & -1.6075900000 \\ \mathrm{~N} & 1.2166900000 & 1.1302900000 & 0.4000100000\end{array}$




\begin{tabular}{|c|c|c|c|}
\hline C & -0.7587200000 & 1.8174700000 & -0.8987400000 \\
\hline C & 2.1898200000 & -0.4323900000 & -1.0097600000 \\
\hline C & 2.3471500000 & 0.3215800000 & 0.1875800000 \\
\hline C & -2.0917800000 & 1.6805700000 & -0.7289400000 \\
\hline C & 3.2046000000 & -1.2750700000 & -1.4316200000 \\
\hline C & 3.5026900000 & 0.2263500000 & 0.9693000000 \\
\hline C & -2.7314700000 & 0.5364500000 & -0.1304900000 \\
\hline C & 4.3668300000 & -1.3700100000 & -0.6555900000 \\
\hline C & 4.5079500000 & -0.6331100000 & 0.5220500000 \\
\hline C & -2.0677000000 & -0.2859300000 & 0.8868200000 \\
\hline C & -4.0284100000 & 0.1965100000 & -0.5389200000 \\
\hline 0 & -0.9998900000 & 0.0646100000 & 1.4172400000 \\
\hline C & -2.7515900000 & -1.4938200000 & 1.3255800000 \\
\hline C & -4.6545600000 & -0.9442200000 & -0.0529300000 \\
\hline C & -4.0051100000 & -1.7987000000 & 0.8652000000 \\
\hline $\mathrm{H}$ & -4.5238500000 & -2.7025200000 & 1.2037400000 \\
\hline $\mathrm{H}$ & -0.3707000000 & 2.7044600000 & -1.4329500000 \\
\hline $\mathrm{H}$ & -2.7679200000 & 2.4596300000 & -1.1194300000 \\
\hline $\mathrm{H}$ & 3.0993900000 & -1.8608300000 & -2.3511400000 \\
\hline $\mathrm{H}$ & 3.6117200000 & 0.8034500000 & 1.8889500000 \\
\hline $\mathrm{H}$ & 5.1745500000 & -2.0388800000 & -0.9728700000 \\
\hline $\mathrm{H}$ & 5.4291000000 & -0.7303800000 & 1.1102500000 \\
\hline $\mathrm{H}$ & -4.5515700000 & 0.8291900000 & -1.2674500000 \\
\hline $\mathrm{H}$ & -2.2305000000 & -2.1302900000 & 2.0446200000 \\
\hline $\mathrm{H}$ & -5.6666400000 & -1.1967500000 & -0.3908900000 \\
\hline C & 0.9892600000 & 1.8558600000 & 1.6202500000 \\
\hline $\mathrm{H}$ & 1.0309900000 & 1.1730600000 & 2.4981800000 \\
\hline $\mathrm{H}$ & 1.7631100000 & 2.6546500000 & 1.7476700000 \\
\hline $\mathrm{H}$ & -0.0116200000 & 2.3261800000 & 1.5998900000 \\
\hline C & -0.0260300000 & -1.3741800000 & -1.7059100000 \\
\hline $\mathrm{H}$ & -0.1111400000 & -1.8469700000 & -0.7192000000 \\
\hline $\mathrm{H}$ & -1.0335900000 & -1.1139300000 & -2.0648700000 \\
\hline $\mathrm{H}$ & 0.4314700000 & -2.0893900000 & -2.4092800000 \\
\hline C & 0.9794600000 & 0.5649400000 & -2.9614400000 \\
\hline $\mathrm{H}$ & 1.4613300000 & -0.1212400000 & -3.6749500000 \\
\hline $\mathrm{H}$ & -0.0088600000 & 0.8469000000 & -3.3556000000 \\
\hline $\mathrm{H}$ & 1.6006200000 & 1.4678900000 & -2.8698500000 \\
\hline
\end{tabular}

40

SO_CCC (SPm)

$\begin{array}{lrrr}C & 0.2581200000 & -0.5274000000 & 0.0963500000 \\ C & 0.0949100000 & -0.7400300000 & 1.6181900000 \\ N & 1.4764400000 & 0.0269600000 & -0.1804700000 \\ C & -0.6775800000 & -0.9620700000 & -0.8425900000\end{array}$




\begin{tabular}{|c|c|c|c|}
\hline C & 1.4201100000 & -0.2106900000 & 2.1302000000 \\
\hline C & 2.1854000000 & 0.2497400000 & 1.0259300000 \\
\hline C & -0.8525500000 & -0.4243000000 & -2.1216000000 \\
\hline C & 1.9289700000 & -0.1068900000 & 3.4144500000 \\
\hline C & 3.4515600000 & 0.8149700000 & 1.1889300000 \\
\hline C & -0.6264400000 & 0.8996000000 & -2.5029600000 \\
\hline C & 3.2015200000 & 0.4528600000 & 3.5857900000 \\
\hline C & 3.9444300000 & 0.9051300000 & 2.4935400000 \\
\hline C & -0.3818700000 & 1.9666400000 & -1.5298100000 \\
\hline C & -0.8494800000 & 1.2527900000 & -3.8730600000 \\
\hline 0 & -0.1872100000 & 1.7407700000 & -0.3166300000 \\
\hline C & -0.4036000000 & 3.3352700000 & -2.0313000000 \\
\hline C & -0.8291100000 & 2.5567300000 & -4.2875500000 \\
\hline C & -0.6088500000 & 3.6060300000 & -3.3498500000 \\
\hline $\mathrm{H}$ & -0.6198100000 & 4.6420800000 & -3.7183100000 \\
\hline $\mathrm{H}$ & -1.3617700000 & -1.7548700000 & -0.5280200000 \\
\hline $\mathrm{H}$ & -1.3470300000 & -1.0850100000 & -2.8665700000 \\
\hline $\mathrm{H}$ & 1.3510100000 & -0.4517500000 & 4.2769700000 \\
\hline $\mathrm{H}$ & 4.0297700000 & 1.1789300000 & 0.3373700000 \\
\hline $\mathrm{H}$ & 3.6169600000 & 0.5467300000 & 4.5943600000 \\
\hline $\mathrm{H}$ & 4.9331800000 & 1.3478600000 & 2.6599000000 \\
\hline $\mathrm{H}$ & -1.0330200000 & 0.4448000000 & -4.5999100000 \\
\hline $\mathrm{H}$ & -0.2302700000 & 4.1271400000 & -1.2991700000 \\
\hline $\mathrm{H}$ & -0.9902700000 & 2.8136500000 & -5.3395700000 \\
\hline C & 2.0557000000 & 0.2471400000 & -1.4791900000 \\
\hline $\mathrm{H}$ & 1.6650700000 & -0.4998700000 & -2.2024800000 \\
\hline $\mathrm{H}$ & 1.8090800000 & 1.2617900000 & -1.8615800000 \\
\hline $\mathrm{H}$ & 3.1620400000 & 0.1430400000 & -1.4262400000 \\
\hline C & -1.0526300000 & 0.1390500000 & 2.0901700000 \\
\hline $\mathrm{H}$ & -2.0044800000 & -0.2406900000 & 1.6883500000 \\
\hline $\mathrm{H}$ & -1.0976300000 & 0.1295900000 & 3.1919800000 \\
\hline $\mathrm{H}$ & -0.9023400000 & 1.1592000000 & 1.7296700000 \\
\hline C & -0.0937400000 & -2.2036800000 & 1.9807400000 \\
\hline $\mathrm{H}$ & -0.1287800000 & -2.3066100000 & 3.0759600000 \\
\hline $\mathrm{H}$ & -1.0388500000 & -2.5830700000 & 1.5628400000 \\
\hline $\mathrm{H}$ & 0.7351300000 & -2.8135800000 & 1.5954900000 \\
\hline
\end{tabular}

40

SO_TCC (SPm)

$\begin{array}{lrrr}C & 0.7319100000 & -0.1747900000 & -0.5925800000 \\ C & 0.2288200000 & 0.6658100000 & 0.6040300000 \\ \text { N } & 1.9675900000 & -0.7381400000 & -0.2855100000 \\ C & 0.0128100000 & -0.6431500000 & -1.6751600000 \\ C & 1.2979200000 & 0.3543400000 & 1.6396200000\end{array}$




\begin{tabular}{|c|c|c|c|}
\hline C & 2.3101600000 & -0.4472200000 & 1.0493200000 \\
\hline C & -1.1922200000 & -0.0929900000 & -2.1675700000 \\
\hline C & 1.4085300000 & 0.7364600000 & 2.9669300000 \\
\hline C & 3.4280200000 & -0.8758600000 & 1.7693700000 \\
\hline C & -1.4378200000 & 1.2511100000 & -2.3884100000 \\
\hline C & 2.5219900000 & 0.3041600000 & 3.6997400000 \\
\hline C & 3.5098900000 & -0.4856200000 & 3.1085500000 \\
\hline C & -0.3298800000 & 2.2119100000 & -2.5398000000 \\
\hline C & -2.7722500000 & 1.6849500000 & -2.6797000000 \\
\hline 0 & 0.8616600000 & 1.8873400000 & -2.4154000000 \\
\hline C & -0.6878900000 & 3.5694300000 & -2.9540000000 \\
\hline C & -3.0386800000 & 2.9778400000 & -3.0324800000 \\
\hline C & -1.9781300000 & 3.9257800000 & -3.1752100000 \\
\hline $\mathrm{H}$ & -2.2391600000 & 4.9465500000 & -3.4853100000 \\
\hline $\mathrm{H}$ & 0.3916400000 & -1.5243800000 & -2.2013500000 \\
\hline $\mathrm{H}$ & -1.9699500000 & -0.8266000000 & -2.4741400000 \\
\hline $\mathrm{H}$ & 0.6418400000 & 1.3607500000 & 3.4368900000 \\
\hline $\mathrm{H}$ & 4.2036100000 & -1.4841100000 & 1.3011300000 \\
\hline $\mathrm{H}$ & 2.6161500000 & 0.5879700000 & 4.7533200000 \\
\hline $\mathrm{H}$ & 4.3674400000 & -0.8140800000 & 3.7078500000 \\
\hline $\mathrm{H}$ & -3.5942400000 & 0.9594100000 & -2.5774000000 \\
\hline $\mathrm{H}$ & 0.1365200000 & 4.2771200000 & -3.0622700000 \\
\hline $\mathrm{H}$ & -4.0661500000 & 3.3107000000 & -3.2141400000 \\
\hline C & 2.8890300000 & -1.2737300000 & -1.2506200000 \\
\hline $\mathrm{H}$ & 2.8476200000 & -2.3915400000 & -1.2671300000 \\
\hline $\mathrm{H}$ & 2.6519700000 & -0.8912300000 & -2.2629400000 \\
\hline $\mathrm{H}$ & 3.9286600000 & -0.9672300000 & -1.0029800000 \\
\hline C & 0.2065000000 & 2.1618200000 & 0.3346600000 \\
\hline $\mathrm{H}$ & -0.6883300000 & 2.4303500000 & -0.2427300000 \\
\hline $\mathrm{H}$ & 0.1839000000 & 2.7025900000 & 1.2962400000 \\
\hline $\mathrm{H}$ & 1.0961100000 & 2.4610100000 & -0.2260200000 \\
\hline C & -1.1234800000 & 0.1390400000 & 1.0602700000 \\
\hline $\mathrm{H}$ & -1.8722600000 & 0.2992400000 & 0.2729400000 \\
\hline $\mathrm{H}$ & -1.0676800000 & -0.9344500000 & 1.2892200000 \\
\hline $\mathrm{H}$ & -1.4380700000 & 0.6779600000 & 1.9672700000 \\
\hline
\end{tabular}

40

SO_TTC (SPm)

$\begin{array}{lrrr}\mathrm{C} & 0.7646100000 & 0.3246300000 & 0.0534900000 \\ \mathrm{C} & 1.4990600000 & 1.0490300000 & -1.0965200000 \\ \mathrm{~N} & 1.6861200000 & -0.0931300000 & 1.0015700000 \\ \mathrm{C} & -0.5839200000 & 0.0564000000 & 0.2040000000 \\ \mathrm{C} & 2.9467200000 & 0.9212600000 & -0.6533700000 \\ \mathrm{C} & 2.9924400000 & 0.2370900000 & 0.5897400000\end{array}$




$\begin{array}{lrrr}\text { C } & -1.5580200000 & 0.4258700000 & -0.7411900000 \\ \mathrm{C} & 4.1230600000 & 1.3334000000 & -1.2586500000 \\ \mathrm{C} & 4.2009800000 & -0.0445900000 & 1.2318100000 \\ \mathrm{C} & -2.8820200000 & 0.0221200000 & -0.6304700000 \\ \mathrm{C} & 5.3384200000 & 1.0540900000 & -0.6213500000 \\ \mathrm{C} & 5.3722500000 & 0.3744000000 & 0.5974600000 \\ \mathrm{C} & -3.3518400000 & -0.8060500000 & 0.4896700000 \\ \mathrm{C} & -3.8183800000 & 0.3924300000 & -1.6515900000 \\ \mathrm{O} & -2.6017200000 & -1.1437900000 & 1.4263300000 \\ \mathrm{C} & -4.7466300000 & -1.2320100000 & 0.4682400000 \\ \mathrm{C} & -5.1153100000 & -0.0395900000 & -1.6137200000 \\ \mathrm{C} & -5.5774300000 & -0.8617600000 & -0.5422900000 \\ \mathrm{H} & -6.6277900000 & -1.1854200000 & -0.5530500000 \\ \mathrm{H} & -0.9387500000 & -0.4965500000 & 1.0726800000 \\ \mathrm{H} & -1.2728200000 & 1.0436400000 & -1.6173000000 \\ \mathrm{H} & 4.1023100000 & 1.8639100000 & -2.2155300000 \\ \mathrm{H} & 4.2273800000 & -0.5725400000 & 2.1875300000 \\ \mathrm{H} & 6.2769700000 & 1.3721100000 & -1.0867500000 \\ \mathrm{H} & 6.3378000000 & 0.1615800000 & 1.0704600000 \\ \mathrm{H} & -3.4674800000 & 1.0274800000 & -2.4804600000 \\ \mathrm{H} & -5.0828600000 & -1.8568100000 & 1.2981200000 \\ \mathrm{H} & -5.8229600000 & 0.2384400000 & -2.4018000000 \\ \mathrm{C} & 1.3588100000 & -0.7543200000 & 2.2392900000 \\ \mathrm{H} & 0.8385100000 & -0.0529400000 & 2.9301600000 \\ \mathrm{H} & 0.6967000000 & -1.6268900000 & 2.0542800000 \\ \mathrm{H} & 2.2808200000 & -1.1171300000 & 2.7411900000 \\ \mathrm{C} & 1.3013200000 & 0.3244900000 & -2.4191300000 \\ \mathrm{H} & 0.2484500000 & 0.3652600000 & -2.7332100000 \\ \mathrm{H} & 1.9208400000 & 0.8008000000 & -3.1949400000 \\ \mathrm{H} & 1.5999600000 & -0.7273900000 & -2.3236600000 \\ \mathrm{C} & 1.0894300000 & 2.5133500000 & -1.1464600000 \\ \mathrm{H} & 1.7391400000 & 3.0551100000 & -1.8510600000 \\ \mathrm{H} & 0.0460500000 & 2.6123200000 & -1.4806500000 \\ & 1.1867100000 & 2.9698900000 & -0.1524100000\end{array}$

40

SO_CTC (SPm)

$\begin{array}{lrrr}C & 0.6429400000 & -0.1947400000 & -0.3418900000 \\ C & 1.5511200000 & 0.7921800000 & -1.1149300000 \\ N & 1.3949400000 & -0.9030400000 & 0.5780100000 \\ C & -0.7103600000 & -0.2501600000 & -0.6186700000 \\ C & 2.8993500000 & 0.5213900000 & -0.4800000000 \\ C & 2.7472500000 & -0.4855400000 & 0.5092100000 \\ C & -1.6674600000 & -1.0354200000 & 0.0487100000\end{array}$




\begin{tabular}{|c|c|c|c|}
\hline C & 4.1455000000 & 1.0797400000 & -0.7163300000 \\
\hline C & 3.8308700000 & -0.9352800000 & 1.2678200000 \\
\hline C & -3.0056400000 & -1.0338300000 & -0.3267100000 \\
\hline C & 5.2356900000 & 0.6297500000 & 0.0380400000 \\
\hline C & 5.0769700000 & -0.3584400000 & 1.0107500000 \\
\hline C & -3.5029000000 & -0.2302700000 & -1.4523700000 \\
\hline C & -3.9383700000 & -1.8405100000 & 0.4054100000 \\
\hline 0 & -2.7526400000 & 0.4978000000 & -2.1320700000 \\
\hline C & -4.9266800000 & -0.3144000000 & -1.7574900000 \\
\hline C & -5.2634100000 & -1.8747400000 & 0.0709700000 \\
\hline C & -5.7566200000 & -1.1022600000 & -1.0232400000 \\
\hline $\mathrm{H}$ & -6.8291800000 & -1.1618700000 & -1.2548900000 \\
\hline $\mathrm{H}$ & -1.0913300000 & 0.3757100000 & -1.4277100000 \\
\hline $\mathrm{H}$ & -1.3808800000 & -1.6609500000 & 0.9138300000 \\
\hline $\mathrm{H}$ & 4.2732700000 & 1.8560500000 & -1.4762900000 \\
\hline $\mathrm{H}$ & 3.7131100000 & -1.7082900000 & 2.0315300000 \\
\hline $\mathrm{H}$ & 6.2264000000 & 1.0624700000 & -0.1343700000 \\
\hline $\mathrm{H}$ & 5.9466800000 & -0.6907700000 & 1.5893200000 \\
\hline $\mathrm{H}$ & -3.5636000000 & -2.4392300000 & 1.2511300000 \\
\hline $\mathrm{H}$ & -5.2856700000 & 0.2827400000 & -2.5978400000 \\
\hline $\mathrm{H}$ & -5.9689200000 & -2.4926600000 & 0.6360800000 \\
\hline C & 0.9559100000 & -1.9899100000 & 1.4085500000 \\
\hline $\mathrm{H}$ & 1.8241700000 & -2.4820200000 & 1.8960700000 \\
\hline C & 1.5584600000 & 0.4428200000 & -2.5951900000 \\
\hline $\mathrm{H}$ & 0.5486600000 & 0.5440200000 & -3.0127500000 \\
\hline $\mathrm{H}$ & 2.2375700000 & 1.1237200000 & -3.1308800000 \\
\hline $\mathrm{H}$ & 1.9036300000 & -0.5897700000 & -2.7440900000 \\
\hline C & 1.1005300000 & 2.2216800000 & -0.8559100000 \\
\hline $\mathrm{H}$ & 1.7749500000 & 2.9204400000 & -1.3744600000 \\
\hline $\mathrm{H}$ & 0.0787300000 & 2.3688800000 & -1.2285700000 \\
\hline $\mathrm{H}$ & 1.1226200000 & 2.4421900000 & 0.2203500000 \\
\hline $\mathrm{H}$ & 0.2736700000 & -1.6259800000 & 2.2140500000 \\
\hline $\mathrm{H}$ & 0.4283200000 & -2.7605100000 & 0.8020600000 \\
\hline
\end{tabular}

40

SO_TCT (SPm)

$\begin{array}{lrrr}\text { C } & 1.0766800000 & -0.1682300000 & -0.7081500000 \\ \mathrm{C} & 0.7174000000 & 0.9160000000 & 0.3256800000 \\ \mathrm{~N} & 1.8909900000 & -1.1209200000 & -0.0852800000 \\ \mathrm{C} & 0.7553100000 & -0.3236600000 & -2.0436000000 \\ \mathrm{C} & 1.5282900000 & 0.4857600000 & 1.5355200000 \\ \mathrm{C} & 2.1763500000 & -0.7403600000 & 1.2436900000 \\ \mathrm{C} & 0.0977700000 & 0.4349000000 & -3.0484400000 \\ \mathrm{C} & 1.6486000000 & 1.0642100000 & 2.7886000000\end{array}$




$\begin{array}{lrrr}\text { C } & 2.9501900000 & -1.4006800000 & 2.2026200000 \\ \text { C } & -0.6655700000 & 1.5855700000 & -3.1516000000 \\ \text { C } & 2.4284100000 & 0.4110800000 & 3.7505300000 \\ \text { C } & 3.0619400000 & -0.7981700000 & 3.4583600000 \\ \text { C } & -0.9339700000 & 1.9695600000 & -4.5617300000 \\ \text { C } & -1.2265500000 & 2.4188700000 & -2.1359400000 \\ \text { O } & -0.5220600000 & 1.2653000000 & -5.4984300000 \\ \text { C } & -1.6571700000 & 3.2100400000 & -4.8232900000 \\ \text { C } & -1.8954300000 & 3.5716600000 & -2.4448000000 \\ \text { C } & -2.0948200000 & 3.9824600000 & -3.7969800000 \\ \text { H } & -2.6269500000 & 4.9253100000 & -3.9798500000 \\ \text { H } & 1.1686400000 & -1.2525700000 & -2.4826200000 \\ \text { H } & 0.2756900000 & -0.0177400000 & -4.0517300000 \\ \text { H } & 1.1442800000 & 2.0067600000 & 3.0189300000 \\ \text { H } & 3.4472700000 & -2.3492200000 & 1.9848500000 \\ \text { H } & 2.5304400000 & 0.8491300000 & 4.7485200000 \\ \text { H } & 3.6608000000 & -1.2891100000 & 4.2340400000 \\ \text { H } & -1.1578500000 & 2.1158900000 & -1.0913700000 \\ \text { H } & -1.8330400000 & 3.4677800000 & -5.8685100000 \\ \text { H } & -2.3103400000 & 4.1988300000 & -1.6469500000 \\ \text { C } & 2.4652800000 & -2.2791600000 & -0.7152300000 \\ \text { H } & 1.6816500000 & -3.0424500000 & -0.9339100000 \\ \text { H } & 2.9708300000 & -2.0020000000 & -1.6688300000 \\ \text { H } & 3.2229100000 & -2.7464400000 & -0.0530200000 \\ \text { C } & 1.1877200000 & 2.2847400000 & -0.1394200000 \\ \text { H } & 0.9656200000 & 2.4465100000 & -1.1975800000 \\ \text { H } & 0.7039100000 & 3.0693600000 & 0.4628600000 \\ \text { H } & 2.2772300000 & 2.3596400000 & -0.0012300000 \\ \text { C } & -0.7534500000 & 0.7650300000 & 0.7092600000 \\ \text { H } & -1.3780800000 & 0.5403700000 & -0.1652500000 \\ \text { H } & -0.8652500000 & -0.0695200000 & 1.4181600000 \\ \text { H } & -1.1068300000 & 1.6855500000 & 1.1988300000 \\ & & & \\ & & & \\ & & \end{array}$

40

SO_CCT (SPm)

$\begin{array}{lrrr}\text { C } & 0.5546300000 & 0.9427200000 & -0.8730800000 \\ \mathrm{C} & 1.7643300000 & 1.8983400000 & -0.7513800000 \\ \mathrm{~N} & 0.8225700000 & -0.2540200000 & -0.2172300000 \\ \mathrm{C} & -0.5671700000 & 1.3133800000 & -1.5771400000 \\ \mathrm{C} & 2.7899700000 & 0.9985800000 & -0.0964600000 \\ \mathrm{C} & 2.1814300000 & -0.2510000000 & 0.1882000000 \\ \mathrm{C} & -1.8489600000 & 0.7004400000 & -1.6055300000 \\ \mathrm{C} & 4.1160100000 & 1.2216300000 & 0.2377100000 \\ \mathrm{C} & 2.8936300000 & -1.2809300000 & 0.8088000000\end{array}$




\begin{tabular}{|c|c|c|c|}
\hline C & -2.5129100000 & 0.1495200000 & -0.5284800000 \\
\hline C & 4.8348100000 & 0.1883700000 & 0.8527300000 \\
\hline C & 4.2287900000 & -1.0357600000 & 1.1388500000 \\
\hline C & -3.8308700000 & -0.4644400000 & -0.8058100000 \\
\hline C & -2.0700300000 & 0.2453600000 & 0.8310500000 \\
\hline 0 & -4.2555400000 & -0.5461300000 & -1.9686000000 \\
\hline C & -4.5848800000 & -0.9875400000 & 0.3297600000 \\
\hline C & -2.8341200000 & -0.2575700000 & 1.8479200000 \\
\hline C & -4.0927900000 & -0.8906100000 & 1.5895500000 \\
\hline $\mathrm{H}$ & -4.6539500000 & -1.2848600000 & 2.4477700000 \\
\hline $\mathrm{H}$ & -0.4689800000 & 2.2044500000 & -2.2084100000 \\
\hline $\mathrm{H}$ & -2.3941700000 & 0.7444100000 & -2.5638900000 \\
\hline $\mathrm{H}$ & 4.5933900000 & 2.1817800000 & 0.0204900000 \\
\hline $\mathrm{H}$ & 2.4245100000 & -2.2412500000 & 1.0292300000 \\
\hline $\mathrm{H}$ & 5.8863300000 & 0.3432200000 & 1.1131100000 \\
\hline $\mathrm{H}$ & 4.8128000000 & -1.8239400000 & 1.6279500000 \\
\hline $\mathrm{H}$ & -1.1297000000 & 0.7657900000 & 1.0461800000 \\
\hline $\mathrm{H}$ & -5.5531400000 & -1.4427200000 & 0.1132700000 \\
\hline $\mathrm{H}$ & -2.5043500000 & -0.1668900000 & 2.8875300000 \\
\hline C & 0.1594200000 & -1.5087700000 & -0.4752400000 \\
\hline $\mathrm{H}$ & -0.9315600000 & -1.3778900000 & -0.5392400000 \\
\hline $\mathrm{H}$ & 0.5192400000 & -1.9626500000 & -1.4277600000 \\
\hline $\mathrm{H}$ & 0.3712300000 & -2.2190900000 & 0.3526000000 \\
\hline C & 2.2416500000 & 2.4056600000 & -2.1025100000 \\
\hline $\mathrm{H}$ & 2.5174600000 & 1.5656700000 & -2.7529700000 \\
\hline $\mathrm{H}$ & 3.1204700000 & 3.0535600000 & -1.9616900000 \\
\hline C & 1.3813600000 & 3.0384300000 & 0.1835200000 \\
\hline $\mathrm{H}$ & 2.2291700000 & 3.7316400000 & 0.2895400000 \\
\hline $\mathrm{H}$ & 0.5201600000 & 3.5877200000 & -0.2221000000 \\
\hline $\mathrm{H}$ & 1.1186900000 & 2.6476500000 & 1.1762900000 \\
\hline $\mathrm{H}$ & 1.4501100000 & 2.9928400000 & -2.5946700000 \\
\hline
\end{tabular}

